See discussions, stats, and author profiles for this publication at: https://www.researchgate.net/publication/334121806

\title{
Attachment Orientations Guide the Transfer of Leadership Judgments: Culture Matters
}

Article in Personality and Social Psychology Bulletin · June 2019 DOI: 10.1177/0146167219865514

\section{CITATIONS}

2 authors:

Jon Gruda

National University of Ireland, Maynooth

16 PUBLICATIONS 6 CITATIONS

SEE PROFILE
READS

411

Konstantinos Kafetsios

University of Crete

89 PUBLICATIONS 1,819 CITATIONS

SEE PROFILE

Some of the authors of this publication are also working on these related projects:

Project Emotion Recognition Accuracy View project

Project Culture and the perception of emotion View project 
RUNNING HEAD: Attachment in leader transfer

Attachment Orientations Guide the Transfer of Leadership Judgments: Culture Matters

Word count: 10,578 


\begin{abstract}
Two experiments tested the role of global and relationship-specific attachment orientations in leader transference, a social-cognitive process in which mental representations of past leaders are associated with the evaluations of new, similar leaders. Individuals scoring higher on anxious attachment were more likely to hold high just treatment expectations of new leaders who were similar to their previous leaders. Conversely, avoidant individuals evaluated new similar leaders low on just treatment expectations and perceived them as less effective. Relationship-specific attachment orientations predicted transfer of behavioral judgments of just treatment, while global attachment orientations predicted transfer of perceived leader effectiveness. These effects were moderated by culture. In two collectivistic cultures (Greece and India), avoidant individuals demonstrated low just treatment expectations of their new similar leader. In an individualistic culture (US), avoidant participants showed high behavioral expectations of their new, similar, leader. The results inform emerging views on relational social-cognitive processes in leader-follower interactions.
\end{abstract}

Keywords: leadership, attachment orientations, culture, social perception 


\section{Attachment Orientations Guide the Transfer of Leadership Judgments: Culture Matters}

In the last three decades, personality and social psychologists have recognized the importance of automatic processes in social perception and behavior. A big part of this social-cognitive approach has stressed the interpersonal mechanisms behind automaticity in social perception: interpersonal goals (Fitzsimons \& Bargh, 2003), the relational self (Andersen \& Chen, 2002), adult attachment information processing (Mikulincer \& Horesh, 1999), and cultural constructions in self-other schemas (Brewer \& Gardner, 1996) to name a few. Research on leader-follower interaction has equally embraced an information-processing approach to understand the automatic cognitive categorization processes influencing leader evaluation (Lord \& Brown, 2003). Yet, despite recent interest in the relational and interpersonal bases of leader perception (Thomas, Martin, Epitropaki, Guillaume, \& Lee, 2013), little research has examined relational antecedents to automatic leader evaluations and even less addressed the social contexts that can shape followers' perceptions of their leaders. The present research examined how key interpersonal social-cognitive schemas, attachment working models of self and significant others, may be associated with the transfer of leader evaluations and how these processes differ as a function of cultural structuring of the self and relationships to others.

Transference is the process by which a perceiver's mental representations of and relationship patterns with a significant other are activated in an encounter with a new person (Andersen \& Chen, 2002). People carry forward behavioral expectations and relationship patterns from one relationship to the next, often unintentionally (Andersen \& Baum, 1994). Given a degree of perceived similarity, mental representations of previous significant others can unconsciously skew perceptions of new individuals (Brumbaugh \& Fraley, 2006; Chen, Andersen, \& Hinkley, 1999). Notably, even a minimal similarity or resemblance is needed 
for the transference process to occur (Chen et al., 1999) as the resemblance is implicit and sometimes even subliminal.

The same transfer process can also apply to vertical, leader-follower relationships. Cognitive representations of previous leaders can be automatically triggered when encountering a new leader, thus influencing individuals' evaluations and expectations of those new leaders (Pierro \& Kruglanski, 2008; Ritter \& Lord, 2007). Transfer of leader mental representations has important implications for leader-follower interaction and followers' self-regulatory processes (Lord, Diefendorff, Schmidt, \& Hall, 2010). Yet, previous research on antecedents to the transfer of leadership expectations is very limited. Adult attachment theory, a key relationship theory on leader-follower interaction (Popper, 2004), can inform such leadership processes. Adult attachment orientations are fit predictors of the transfer of expectations between similarly perceived interaction partners, since they encompass mental representations of self and significant others, which are carried forward into new relationships (Brumbaugh \& Fraley, 2006, 2007). Moreover, the cultural context can shape the relative emphasis attachment orientations place on self and significant others (Friedman et al., 2010; Zhao et al., 2018) and can likely influence the respective transference of mental representations.

\section{Attachment Orientations and Transference Processes}

As a key framework for interpersonal functioning, adult attachment orientations and the corresponding mental representations of self and significant others (internal working models) are the product of early socialization experiences (Mikulincer \& Horesh, 1999). Over time, and as a result of interactions with significant others, individuals develop a dominant attachment orientation that differentiates and explains individuals' reactions and behavior during encounters with new interaction partners (Brumbaugh \& Fraley, 2006; Collins \& Read, 1994). An avoidant attachment orientation generally results from experiences of 
interactions with unresponsive caregivers. The corresponding working model of avoidant attachment involves a negative view of and distance from others, with an emphasis and focus on the self and deactivation of the attachment system by suppressing and limiting accessibility to emotional memories and thoughts (Mikulincer \& Shaver, 2007). Conversely, an anxious attachment orientation and a corresponding working model where others are viewed more positively than the self typically leads individuals to experience a strong need for intimacy and emotional closeness with important others (Popper \& Amit, 2009). Therefore, persons with an anxious attachment orientation adopt hyperactivating cognitive and behavioral strategies such as seeking increased proximity to their attachment figure (Mikulincer \& Shaver, 2007).

When attachment and related mental representations are activated, individuals can recreate past relationship patterns in new relationships (Collins \& Read, 1994). Insecure attachment orientations can help explain why some people are more likely than others to transfer previous mental representations of others and relationship patterns onto new interaction partners (Brumbaugh \& Fraley, 2006, 2007). Engaging in this anchoring effect of previous significant others and relationships, and a subsequent transfer of mental representations from one relationship to another, leads to the stability of attachment orientations across relationships (Brumbaugh \& Fraley, 2007; Fraley \& Brumbaugh, 2004). The present studies experimentally tested associations between attachment orientations and the transfer of leader expectations from one leader to another.

\section{Attachment Orientations and Leader Perception}

Attachment is a key framework for understanding work relationships and leaderfollower interaction in particular (Davidovitz, Mikulincer, Shaver, Izsak, \& Popper, 2007). There has been an explosion of interest in applying attachment theory to leader-follower interaction (e.g., Mayseless \& Popper, 2018), yet little research has looked at social-cognitive 
facets of attachment perceptions for leader-follower interaction. Examining whether and how attachment working models are associated with the transference processes can inform both relationship and leadership theories.

In leader-follower interaction, followers not only perceive and observe leaders' behavior but also learn how the leader reacts in a variety of situations. Employees base their predictions of future leader behavior subconsciously on previous encounters with their leader (Ritter \& Lord, 2007). Assuming that perceivers' mental representations of a previous leader are salient enough to categorize that leader as a significant other, such mental representations could activate followers' self-concept (Lord \& Brown, 2003). The relationship between "self and other" is then likely transferred to new encounters (Andersen \& Glassman, 1996; Hinkley \& Andersen, 1996). Attachment orientations have been shown to affect the transference of such mental representations and relationship patterns between partners (Brumbaugh \& Fraley, 2006, 2007), yet no previous work has tested links between attachment orientations and the transfer of leader expectations.

We based our hypotheses upon the assumption that individuals' working models of a current or existing attachment figure are activated by a degree of similarity between the mental representation of the specific interaction partner in mind and a new interaction partner (Brumbaugh \& Fraley, 2007). In such instances, persons are likely to transfer mental representations and relational expectations to a new relationship target. We expect this transfer to occur whenever there is a large enough overlap (similarity) between an existing mental representation of a current or previous interaction partner and a description of the new relationship target (Ritter and Lord, 2007). We began our research by aiming to establish that the transference process from one leader to another is possible using a new computerized experimental method adapted from Ritter and Lord (2007). In addition to examining 
expectations of just treatment and positive and negative affect, we also introduced leader effectiveness, a more cognitive measure of leadership.

H1. Leader similarity will moderate the transfer of mental representations of one's previous to a new leader, such that previous leader just treatment and leader effectiveness will be strongly related to the new leader just treatment and leader effectiveness expectations in a new similar leader in comparison to a new less similar leader.

We did not form specific hypotheses regarding the transfer of affect given that Ritter and Lord (2007) did not find a transfer of positive and negative affect in the evaluation of new leaders who exhibited a certain degree of similarity to previous leaders.

\section{Attachment orientations as moderators to the leader transfer process}

The core of our hypotheses was that perceived similarity will activate avoidant and anxious attachment working models, which in turn would shape new leader perceptions. We expected that higher attachment anxiety and avoidance may be associated with new leader perceptions as a function of perceived similarity between previous and new leader descriptions. At the core of the leader-follower transfer process is the transfer of significant other mental representations. Anxious and avoidant attachment orientations involve working models of significant others, the self, and the relationship between self and significant others that result from experiences in interactions with significant others. When activated, avoidant and anxious working models tend to trigger different self-other perceptual processes, which also involve secondary strategies of emotion regulation that encompass projective mechanisms associated with the respective working models (Mikulincer, 1998). Persons high on avoidant attachment tend to hold a more negative model of significant others and an unrealistically positive model of the self (Pietromonaco \& Barrett, 2000). Thus, an avoidant working model involves perceptions of significant others as different from one's self as a 
result of a defensive regulation that favors distance of the self from significant others and a focus on the self (Mikulincer, 1998; Mikulincer, Orbach, \& Iavnieli, 1998). An anxious attachment working model, on the other hand, involves positive models of significant others, in line with defensive strategies that connect others with the self (Mikulincer \& Horesh, 1999). Efforts to exaggerate personal weaknesses and bring out other people's attention and concern for the self are in keeping with an overarching goal of maintaining connectedness with others and a heightened similarity with others (Mikulincer \& Shaver, 2007).

Therefore, in so far as the similarity between current and previous leaders would activate attachment working models, anxious compared to less anxious participants are anticipated to evaluate a new similar leader higher on just-treatment expectations and leader effectiveness. Avoidant individuals, on the other hand, are expected to evaluate new similar leaders lower in just treatment and leadership effectiveness, due to defenses that favor distance of the self from significant others. The predictions are as follows:

H2a. Leader similarity will moderate the negative relationship between avoidant attachment orientation and just-treatment expectations and perceived leader effectiveness of a new leader. Higher avoidant attachment will be associated with lower leader-just-treatment expectations and perceived leader effectiveness, in the condition of high (vs. low) similarity to the previous leader.

$H 2 b$. Leader similarity will moderate the positive relationship between anxious attachment orientation and the evaluation of a new leader in terms of just-treatment expectations and leader effectiveness. Higher anxious attachment will be associated with higher just treatment expectations and leader effectiveness, particularly in the condition of high (vs. low) similarity activation to the previous leader.

\section{Effects of global and relationship-specific working models}


An important question in attachment research concerns whether working models are trait-like in the way they function (i.e., applied to a broad array of interpersonal situations and interaction partners) or whether they are sensitive to context (i.e., applied as a function of the perceived closeness with the person with whom one interacts; see Baldwin, Keelan, Fehr, Enns, \& Koh-Rangarajoo, 1996; Pierce \& Lydon, 2001). Global attachment orientations involve chronically accessible working models, with cognitive and affective properties based on experiences in previous relationships. Global attachment working models are applied to new relationships in general and describe how individuals typically perceive close others and feel toward those relationships. In most previous research, it is global or chronic attachment models that were measured.

Over time, individuals develop numerous relationships with significant others, therefore creating and forming person-specific and relationship-specific working models of self and other (Klohnen, Weller, Luo, \& Choe, 2005). Relationship-specific attachment working models are considered nested within global models and concern emotions and behaviors in specific relationship contexts (Overall, Fletcher, \& Friesen, 2003).

Previous research has found that both global and relationship-specific working models can predict the expectations of new interaction partners. Even when there is limited overlap between the focal person (the previous interaction partner) and the target (a new interaction partner), mental representations or working models of attachment from previous interaction partners guide individuals in their relational behavior and feelings toward the target person (Brumbaugh \& Fraley, 2006). However, Brumbaugh \& Fraley (2006) have also observed some differentiation in the way global and relationship-specific working models affected transference. Specifically, they observed that "relationship-specific avoidance is more influential than global avoidance on the difference in participants' feelings" (p. 557). Equally, Overall et al. (2003) maintain that "which model or level of attachment representations is 
primarily accessed will depend on the specificity of the relationship context... including current goals, emotions and past experiences" (p. 1491).

Based on the above findings we, therefore, expected that the activated global and relationship-specific attachment working models would predict evaluations of new leaders, depending on the interpersonal-emotional context of the evaluation. Highly emotional judgments, such as emotion toward someone or expectations of just treatment from someone, are more likely to be predicted by the recollection and activation of relationship-specific attachment since relationship-specific working models are more closely associated with episodic memories of emotion events (Overall et al., 2003). On the other hand, we expected more generic judgments that do not evoke an intimate or emotional response by participants, such as judgments of someone's competence or effectiveness, to be predicted by the activation of global attachment working models. Therefore, we hypothesized that:

$H 3 a$. Relationship-specific attachment orientations are more likely to be activated and associated with subsequent judgments of new leaders when participants are asked to indicate ratings of a largely emotional measure, such as just treatment expectations. Consequently, individuals' relationship-specific anxious or avoidant attachment orientations to the previous leader are likely associated with just-treatment evaluations of the new leader in line with $\mathrm{H} 2$.

$H 3 b$. Global attachment orientations are more likely to be activated and associated with subsequent judgments of new leaders when participants are asked to indicate ratings of a largely cognitive measure, such as perceived leader effectiveness. Consequently, individuals' global anxious or avoidant attachment orientation are likely associated with perceived leader effectiveness evaluations of the new leader in line with $\mathrm{H} 2$.

\section{Culture as a moderator}


RUNNING HEAD: Attachment in leader transfer

Recent evidence suggests that culture can modulate followers' implicit perceptions of leaders' attachment orientations (Kafetsios, Athanasiadou, \& Dimou, 2014). We reasoned that central cultural orientations in terms of individualism (i.e., cultural proclivity to view the self as influenced by personal goals and values) versus collectivism (i.e., cultural tendency to see the self as connected with and influenced by others, especially close others) would influence the transference process, since these cultural orientations overlap strongly with attachment models of self and other (Agishtein \& Brumbaugh, 2013).

A collectivistic culture encourages more other-oriented thinking and behavior since the emphasis is on relationships and obligations to others. Therefore, in collectivistic cultures, a model of other is fostered in comparison to focus on the self. Accordingly, a higher prevalence of anxious attachment has been found in more collectivistic cultures (Cheng \& Kwan, 2008) and a combination of higher model of others with lower model of self scores (Schmitt et al., 2004). Anxious persons are more satisfied in their relationships in a more collectivistic culture, such as Mexico, than in a more individualistic one, such as the United States (Friedman et al., 2010), likely due to an alignment with collectivistic norms and otheroriented behavior and thinking.

Conversely, in individualistic cultures, a combination of higher models of other and lower model of the self, characteristic of avoidant attachment, is found to be more prevalent given existing individualistic norms of relating (Schmitt et al., 2004). Accordingly, avoidant individuals in collectivistic cultures experience more relationship problems than avoidant persons in individualistic cultures (Friedman et al., 2010) as they would have to engage in a higher degree of other-oriented behavior such as self-disclosure and care-taking behaviors (Mikulincer \& Nachshon, 1991), with which they are uncomfortable.

We, therefore, expected that a culturally prescribed focus on others in a collectivistic society will amplify the positive expected relationship between anxious attachment and new 
leader perception and the negative expected relationship between avoidant attachment and leader expectations. Conversely, in individualistic cultures, such as the US, we expected that a culturally prescribed focus on the self would attenuate those expected relationships resulting in a weak or no relationship between anxious and avoidant attachment and leader perception. We examined these possible cultural differences in an exploratory manner in Study 2.

\section{Overview of Studies}

In order to manipulate new leader similarity to the previous leader, we adapted the idiographic process used in past transference research (Hinkley \& Andersen, 1996; Ritter \& Lord, 2007) for use with online samples. As we presented participants with a list of traits, rather than having them describe their leader in their own words, our experiment differs slightly from the design of Ritter \& Lord (2007). Therefore, we conducted a pre-test without predictors in order to determine replicability to previous results. Subsequently, Study 1 tested attachment orientations associations with leader transference using a sample of working professionals. Study 2 examined the association of culture and the attachment - leader transference with additional data from the United States and India.

\section{Pre-Test}

\section{Sample and Procedure}

We recruited 258 participants using Amazon Mechanical Turk (Amazon MTurk). Two hundred and eleven participants completed both parts of our experiment: 93 women and 118 men, 21 to 74 years of age $\left(M_{\mathrm{age}}=37.5, S D=11.14\right)$ and average work experience of 16.88 years $(S D=11.18)$. Most $(58.29 \%)$ were in an employee position without supervisory responsibilities and all had completed either high school or equivalent. 
RUNNING HEAD: Attachment in leader transfer

Pretest-Time 1 (T1). Participants were asked to select and rank attributes to describe their current supervisor. Unemployed participants were asked to think of their most recent supervisor. This being the first online experimental study testing leader transference, we made some slight modifications to Andersen's original methodology as applied by Ritter and Lord (2007). Instead of writing down twenty attributes describing their leader from other leaders, participants were shown 16 items from a list of 200 attributes that were previously scored on importance when applied to supervisors by a separate, large-participant sample (Jarymowicz, 1992). These items were presented at random, but evenly (positive, negative and neutral words were shown equally in frequency). From these sixteen items, participants were asked to select and rank the ten attributes that were most definitional in describing their leader. In case participants could not choose from the presented list, they were free to drop out of the survey, which occurred only once. Directly following the ranking task, participants filled out three scales on their relationship with the described leader: positive and negative affect (Watson \& Clark, 1991), interactional justice/just treatment (Colquitt, 2001), and leader effectiveness. Participants also completed standard demographic information, were thanked for their participation and informed about a follow-up study a week later.

Pre-Test - Time 2. We randomly assigned participants to either a high $(\mathrm{n}=103)$ or low similarity $(\mathrm{n}=108)$ new leader condition. Both conditions were preceded by a short vignette, asking participants to imagine they had been assigned a new direct supervisor. and that they were heading to their colleague's office to ask about the new supervisor: "Your colleague tells you that the new head of the department and your new direct supervisor is best described with the following attributes." This descriptive text was followed by a list of seven attributes describing their new leader.

As in Ritter and Lord (2007) we composed an individualized new leader "profile" based on each participant's previous responses and the condition to which they were 
randomly assigned. In the higher similarity condition, we presented participants with four idiographic leader attributes from those pre-tested in Phase 1 and ranked $2-5^{1}$. The highest rank equals the highest number of points given to that attribute by a separate sample of participants (Anderson, 1968). The six most neutral attributes were selected as our neutral descriptor items based on the median of all ranked attributes. We then randomly assigned three of these six neutral, or filler attributes, to each participant's new leader profile. In summary, participants in the higher similarity condition were randomly presented with four idiographic attributes and three neutral attributes.

In the low similarity condition, participants were presented with four leader attributes from another randomly chosen participant (attributes listed and ranked 2-5). The low similarity leader condition also included three neutral items selected from the same list of six neutral leader attributes, based on Jarymowicz (1992). We ensured that none of the participants' selected attributes overlapped with the attributes of the other randomly selected participant. If this instance occurred, we replaced the corresponding duplicate neutral item with another neutral item. Participants were told to take a moment to read through these attributes and let them sink in. Neutral items in both conditions were drawn at random from the same list of six neutral attributes.

In summary, participants in the low similarity condition were presented with four non-idiographic attributes, as well as three neutral attributes. All attributes were shuffled and presented at random. Participants then filled out measures of just-treatment expectations, leader effectiveness as well as positive and negative affect about their new leader and their expected relationship with them.

\section{Manipulation Check}

\footnotetext{
${ }^{1}$ The Ritter and Lord (2007) study included twenty descriptors at T1 and eight idiographic leader descriptors (those listed and ranked 4-11 in Phase 1).
} 
RUNNING HEAD: Attachment in leader transfer

As a manipulation check at the end of the online experiment, we asked participants to indicate how similar the leader described at Time 2 seemed in comparison to the leader participants described at Time 1 . A significant difference $(t=-3.35, p=.001)$ between scores of participants in the higher $(M=2.41, S D=1.02)$ and low similarity leader condition $(M=$ $1.96, S D=.91)$ emerged. These results suggest that participants could somewhat recall and were aware that the described leader was either similar or less similar compared to the leader described at Pre-test T1.

\section{Measures}

Just Treatment perceptions were assessed with the interactional justice scale (Colquitt \& Rodell, 2011) modified appropriately for inclusion at T1 and T2. At T1, the scale included the following items: "Has your supervisor treated you in a polite manner?"; "Has your supervisor treated you with dignity?"; "Has your supervisor treated you with respect?"; and "Has your supervisor refrained from improper remarks or comments?". At T2, the items on this scale were slightly reworded to reflect just treatment expectations of the new leader. The scale included the following items: "Do you expect this new supervisor to treat you in a polite manner?"; Do you expect this new supervisor to treat you with dignity?"; "Do you expect this new supervisor to treat you with respect?"; and "Do you expect this new supervisor to refrain from improper remarks or comments?”.

Leadership Effectiveness was measured using a simple one-item question: "This supervisor is very effective as a leader" (T1). At T2, the item was slightly reworded: "My new supervisor seems very effective as a leader".

Positive and Negative Affect (PA/NA) were measured using the Positive and Negative Affect Schedule (Watson \& Clark, 1991). All scale responses were rated on a fivepoint scale. All statistical analyses were performed using STATA 12 (StataCorp., 2011). 
RUNNING HEAD: Attachment in leader transfer

\section{Results}

Correlations between study variables are reported in Table 1.

Insert Table 1 about here

For statistical efficiency — in order to estimate standard errors more precisely—we estimated a structural equations model, in which all dependent variables were modeled as outcomes simultaneously. The model mimics the model presented in Ritter and Lord (2007), but it lacks the justice latent factor. Instead, it accounts for only interactional justice (Colquitt, 2001). Our results reported in Figure 1, mirrored those of Ritter and Lord (2007), in that, just-treatment expectations were more likely to be transferred to a new leader if the new leader was highly similar to participants' current leader. Parameter estimates for just-treatment expectations do differ significantly across the non-similar and similar leader groups: $\chi^{2}(1)=7.03, p<.01$.

Insert Figure 1 about here

Likewise, we found that leader-effectiveness expectations were more likely to be transferred to a new leader if the new leader was highly similar to participants' current or previous leader. Parameter estimates did differ significantly across groups: $\chi^{2}(1)=16.00, p<$ .001. Regarding positive and negative affect, parameter estimates were significant, both in the high and the low similarity leader condition. Parameter estimates for negative and positive affect did not significantly differ across groups: $\chi^{2}(1)=.03$, ns, and $\chi^{2}(1)=3.56$, ns, respectively. This result suggests that positive and negative affect relate positively to both a previous and a new leader, regardless of the new leader's similarity to the previous leader (Ritter \& Lord, 2007). 


\section{Brief Discussion}

We utilized a standard experimental design, modified for a computerized experimental study, confirming our hypotheses with regard to the transfer of expectations for leadership effectiveness and just treatment (H1). In the pre-test, we successfully replicated results reported by Ritter and Lord (2007) with regard to just-treatment expectations and positive and negative affect, as well as leader-effectiveness expectations. Significant other (leader) transference was evidenced in this pre-test since an overlap (similarity) between an existing mental representation of a current or previous leader influenced the description of a new leader target. Since parameter estimates for positive and negative affect did not differ significantly between the two manipulation conditions, we no longer include positive and negative affect in the following studies.

\section{Study 1}

By using our pre-tested experimental setup, we were in a position to examine, a) whether working models of attachment impact the transfer of evaluations from previous leaders to new similar leaders (H2) and b) differences between global and relationshipspecific attachment orientations (H3). In Study 1 we conducted the experiment using a traditional participant pool of working professionals in Greece.

Sample and Procedure. In total, 130 Greek working professionals participated in this study. An a priory power analysis using G*Power (Faul, Erdfelder, Lang, \& Buchner, 2007) showed that in order to detect a mid-sized effect (e.g., $f^{2}=12$ ) a minimum sample size of $N=$ 120 would be needed for a desired statistical power level $(>=.80)$. Participants were contacted through executive seminars, lifelong learning classes, and a mailing list of previous students in a large university in Greece. All questionnaire text was translated from English into Greek using back-translation techniques (Brislin, 1970). Previously used and translated 
versions of scales were used whenever available. Having passed mandatory attention checks (Mason \& Suri, 2012), our final sample of 95 participants contained $71.58 \%$ females and $28.42 \%$ males, ranging from $19-78$ years of age $(M=33.93, S D=12.39)$, with an average work experience of 10.79 years $(S D=9.82)$. Most participants $(77.89 \%)$ indicated they were employees with no supervisory responsibilities. Participants mostly (53.68\%) worked in management, professional, services, sales, and office jobs, whereas $34.74 \%$ selected 'other' and $1.05 \%$ were unemployed.

Study 1 - Time 1. Participants completed an informed consent form followed by measures of global attachment (Richards \& Schat, 2011), Big-Five personality characteristics (Goldberg et al., 2006) and trait anxiety (Spielberger, 1983).

Participants were again shown randomly, but evenly, a selected sample of sixteen traits, based on a list of previously tested descriptive traits. The trait list in this study included 180 of the previous 200 items, due to an overlap between several traits once translated into Greek (back-to-back translation). As in the pre-test, participants selected and ranked the ten attributes that were most definitional in describing their leader. Participants could leave the survey if not able to choose from the presented list. None did. Next, participants completed measures of relationship-specific attachment to their leader (Fraley, Heffernan, Vicary, \& Brumbaugh, 2011), just treatment (T1), and leader effectiveness (T1). Participants also indicated how important the relationship to their leader is to them, as well as how much contact they have with their leader. Finally, participants completed demographics, were thanked for their participation and were informed about a follow-up study one week later.

Study 1 - Time 2. As in the pre-test, participants were randomly assigned to a new highly $(n=44)$ or less similar $(n=51)$ leader description. Both conditions were preceded by the same short vignette presented in the pre-test, followed by a list of seven attributes describing their new leader. All attributes were randomly presented. Participants were told to 
take a moment to go through these attributes and were asked to indicate their expectations of their new leader with regard to just treatment and leadership effectiveness. Participants then completed leadership effectiveness and just-treatment expectations scales with regard to the just-presented leader, were fully debriefed and thanked for their help.

\section{Measures}

Measures of just treatment (Colquitt, 2001) and perceived leader effectiveness were identical to those listed in the pre-test, as outlined for $\mathrm{T} 1$ and $\mathrm{T} 2$, respectively. We also examined several individual characteristics at $\mathrm{T} 1$.

Global Attachment Orientation was assessed using Richards and Schat (2011) adaptation of Brennan, Clark, and Shaver (1998) Experience in Close Relationships scale (ECR). The ECR comprises 36 items on two subscales: anxious attachment (18 items) and avoidant attachment (18 items). Responses were rated on a seven-point scale $(1=$ strongly disagree; 7 = strongly agree) .

Personality. We used the mini-IPIP scale (International Personality Item Pool; Goldberg et al., 2006) to control for Big-5 personality traits, reported on a five-point scale (1 $=$ very inaccurate; $5=$ very accurate) .

Trait Anxiety was measured with ten items from the Spielberger (1983) inventory, rated on a four-point scale $(1=$ almost never $4=$ almost always $)$.

After completing the leader evaluation task, we administered the following measures to participants. All the following measures below were assessed at T1.

Relationship-Specific Attachment was measured using the scale proposed in Fraley, Hudson, Heffernan, and Segal (2015) at T1. It includes nine items, which are asked with regard to a relationship to a specific person, in this case, participants' current leader. Items 
RUNNING HEAD: Attachment in leader transfer

included statements such as, "It helps to turn to this person in times of need". Responses were rated on a seven-point scale $(1=$ strongly disagree; 7 = strongly agree $)$.

Importance of Relationship was assessed using the following item: "My supervisor is important to me", rated on a five-point scale $(1=$ not at all; $5=$ extremely $)$.

Daily Contact with participants' leader (T1) was assessed using the following: "On a daily basis, I have to my supervisor", rated on a five-point scale $(1=$ no or barely any contact; $5=$ a lot of contact).

\section{Results}

Correlations and basic psychometric characteristics of variables are reported in Table 2.

Insert Table 2 about here

Just-Treatment Expectations. Using ordinary least squares (OLS) regressions with a heteroscedastic-robust estimate of the variance, we regressed the degree of just-treatment expectations attributed to the described leader (T2) onto the manipulated leader similarity condition, relationship-specific anxious and avoidant attachment (T1), and their interaction, controlling for trait anxiety, relationship importance and daily contact in the final model (Table 3, Model 3). Results differed only slightly when controlling for the Big Five personality traits, relationship importance, and daily contact, and can be obtained from the authors.

Insert Table 3 about here

We found that relationship-specific (RS) attachment predicted just-treatment expectations, in line with H3a (see Table 3). Namely, the interaction between relationshipspecific anxious attachment and manipulated similarity condition was a significant predictor 
RUNNING HEAD: Attachment in leader transfer

of just treatment expectations (Table 3, Model 3; $b=.23, S E=.12, p<.05$ ). The interaction is depicted in Figure 2. A simple slopes test found that anxious attachment (of $-1 /+1 \mathrm{SD}$ ) in the higher similarity condition was significant ( simple slope $=.35, t=4.15, p<.000$ ). Therefore, in line with $\mathrm{H} 2 \mathrm{a}$, for a new, highly similar leader, as relationship-specific anxious attachment increased, there was also a significant increase in the ratings of just-treatment expectations. Yet, ratings of just treatment did not significantly increase as a function of relationship-specific anxious attachment in the low similarity condition (simple slope $=.11, t$ $=1.11, p=.27)$.

Insert Figure 2 about here

We also found a significant interaction between relationship-specific avoidant attachment and the manipulated similarity condition in predicting just treatment expectations (H2b). In this case, the interaction was negative (Table 3, Model 3; $b=-.57, S E=.26, p<$ .05). A plot of the interaction is presented in Figure 3. A simple slopes test of RS avoidant attachment (of $-1 /+1 \mathrm{SD}$ ) in the high leader similarity condition was significant (simple slope $=-.31, t=-2.67, p<.01)$. Therefore, as relationship-specific avoidant attachment increased, ratings of just-treatment expectations for new, highly similar leaders significantly decreased; however, in the low leader similarity condition there was no significant slope as a function of relationship-specific avoidant attachment (simple slope $=.31, t=1.36, p=.18$ ).

Insert Figure 3 about here

Finally, we also found a significant three-way interaction between the manipulated leader similarity condition and the interaction between RS anxiety and RS avoidance (Table 3, Model $3 ; b=.18 ; S E=.08, p<.05)$. Decomposing the interaction using marginal 
comparison demonstrated that individuals with high RS-avoidant orientations attributed the lowest just-treatment expectations to new, more similar leaders $(M=2.83, S E=.19 ; t=$ 14.85) compared to anxious $(M=3.09, S E=.25, t=12.34)$, and secure participants $(M=$ $3.18, S E=.22, t=14.45)$.

Insert Table 4 about here

Leader Effectiveness Expectations. Leader-effectiveness expectations were only significantly predicted by global attachment orientations (see Table 4). Results are in line with H3b. Specifically, we observed a marginally significant interaction between anxious attachment and the leader similarity condition (Table 4 , Model $3 ; b=.72, S E=.36, p=.052$ ). A simple slopes analysis revealed that participants higher in global anxious attachment had higher leader-effectiveness expectations in the high leader similarity condition compared to those with lower anxious attachment orientations ( simple slope $=.62, t=2.48, p=.014)$. A significant slope was not observed in the low leader similarity condition (simple slope $=-.09$, $t=-.43, p=.67)$.

With regard to global avoidant attachment, our regression model depicted a negative relationship with leader effectiveness at $\mathrm{T} 2$ as a function of manipulated leader similarity condition (Table 4, Model 3; $b=-1.0 ; S E=.49, p<.05$ ). The interaction is depicted in Figure 4. Higher global avoidant attachment was associated with lower leader effectiveness evaluations in the high leader similarity condition; however, ratings of leader effectiveness decreased as a function of global avoidance in the low leader similarity condition. A simple slope analysis showed that this slope was not significant ( simple slope $=-.41, t=-1.46, p=$ $.145)$.

Insert Figure 4 about here 
Finally, there was a three-way interaction between leader similarity condition and the two attachment orientations, suggesting that global fearful attachment showed a significant link to the dependent variable, as a function of the leader similarity manipulation (Table 4, Model 3; $b=.44, S E=.17, p=.01)$. Closer observation showed that people with higher global avoidant attachment orientations attributed the lowest leader-effectiveness expectations to new, similar leaders $(M=2.68, S E=.37, t=7.73)$ compared to anxious $(M=$ 3.05, $S E=.30, t=10.16)$, fearful $(M=3.97, S E=.26, t=15.27)$ and securely attached participants $(M=3.20, S E=.26, t=12.25)$.

\section{Brief Discussion}

Study 1 tested the relationship between attachment working models and the leader transfer using the pre-tested online experimental set-up. Leader similarity was a consistent moderator of the relationship between insecure attachment orientations, and just-treatment and perceived leader effectiveness expectations of a new leader. When mental representations of a previous leader were primed (through manipulating similar-leader characteristics), higher relationship-specific avoidant attachment to that previous leader was associated with lower just-treatment expectations of a new leader $(\mathrm{H} 2 \mathrm{a})$ whereas higher relationship-specific anxious attachment led to higher just-treatment expectations of the new leader $(\mathrm{H} 2 \mathrm{~b})$. In the primed similar-leader condition only, higher global avoidant attachment was also associated with lower new leader effectiveness perceptions (H2a), and anxious attachment was associated with higher effectiveness perceptions of a new similar leader (H2b). Moreover, a combination of higher anxiety and avoidance (characteristics of the fearful attachment orientation) was associated with higher new leader perceptions.

These findings clearly demonstrate that leader mental representations (priming leader similarity traits) activated attachment working models which in turn relate to leader 
RUNNING HEAD: Attachment in leader transfer

perceptions in theoretically anticipated ways. Moreover, initial relationship-specific attachment working models seem to be a better predictor of emotional expectations from the new leader, such as expectations of just treatment $(\mathrm{H} 3 \mathrm{a})$, whereas global attachment measures predicted the transfer of perceived expectations of leadership effectiveness (H3b). These findings point to differences in the transfer of leadership perceptions between persons with anxious and avoidant attachment orientations. Overall, the findings confirm expectations regarding the different regulatory and perceptual parts of anxious and avoidant adult attachment organization (Mikulincer \& Horesh, 1999) and extend those previous findings to the leadership field.

\section{Study 2}

Study 1 provided good insights on the underlying mechanics of transference using attachment orientations as predictors. To understand whether culture could be a factor to consider in the transfer of behavioral expectations, we compared the presented Greek sample to samples in the United States (US) and in India, based on their degree of individualism vs. collectivism. United States was chosen as the comparison country because the US exhibits one of the highest degrees of individualism (Hofstede, 2001). India was chosen for several reasons. First, most studies examining attachment distributions across countries have focused on East Asian collectivistic countries, whereas attachment data in India are still lacking (Van Ijzendoorn \& Sagi-Schwartz, 1999). In the Indian population, the model of other is negatively correlated with self-esteem, whereas for other collectivistic countries (e.g., Greece, Turkey, and Japan) both models of the self and other correlate positively with self-esteem (Schmitt et al., 2004). People from India also usually score lower on anxious attachment than other collectivistic countries (Agishtein \& Brumbaugh, 2013), such as Greece.

\section{Sample and Procedure}


RUNNING HEAD: Attachment in leader transfer

The procedure remained the same as described in Study 1. The sample consisted of working professionals recruited online using Amazon MTurk in both the US and in India, as well as our Greek population sample from Study 1. The final US sample included 137 participants $(51.80 \%$ female $)$, with a $M_{\text {age }}=37.37(S D=11.74)$ and $M_{\text {work }}=16.15$ years of experience $(S D=10.77)$. Approximately half of our US participants were employees with no supervisory role $(55.40 \%)$.

In the Indian sample, we had to control for language comprehension in addition to ensuring that participants did not answer questions randomly. In total, 144 workers completed both parts of the experiment yielding a final sample of 109 useful responses. Participants had a $M_{\text {age }}=34.04(S D=9.81)$ and $M_{\text {work }}=9.15$ years of experience $(S D=7.57)$. Only $12.84 \%$ of our recruited Indian workers were employees with no supervisory role.

First, we assessed each culture separately. This process was carried out in order to outline the effect of our predictors using the low similarity leader condition as the baseline. We included the findings in the Greek sample in the presented results once again to allow for easier side-by-side comparison. Secondly, we included all three groups in the same model; the US sample served as the baseline, as the US scores highest on individualism (Hofstede, 2001).

\section{Measures}

Displayed traits were chosen from the same list (180 traits) as described in Study 1. Measures were identical to those outlined in Study 1.

\section{Results}

As expected, mean levels of attachment anxiety were higher in the two collectivistic cultures, India and Greece, compared to the US. Avoidance was also higher in the US than in 
the other two countries. An overview of the main statistical information is provided in Table 5 , broken down by culture.

Insert Table 5 about here

Correlation and main psychometrics of all variables of interest are provided in Tables 6 and 7 for the US and the Indian samples, respectively.

Insert Tables $6 \& 7$ about here

In terms of just-treatment expectations, we compared the Greek and Indian samples against the American sample. In the case of leader-effectiveness evaluations, the relationshipspecific version of the scale was replaced with the global attachment orientation ratings. Again, results differed only slightly when controlling for Big-Five personality dimensions and can be obtained from the authors.

Insert Table 8 about here

Results from the regression analyses showed significant differences between the three samples. With the US sample as a baseline, in the Greek sample in the similar-leader condition, relationship-specific avoidant attachment related negatively to $\mathrm{T} 2$ just-treatment expectations (Table 8 , Model $3 ; b=-.81, S E=.33, p<.05$ ). The same pattern was found in the Indian sample (Table 8, Model 3; $b=-.91, S E=.43, p<.05$ ). Given these results, we examined whether the partial coefficients of the fit measures differed across the three groups. Coefficients were significantly different (see Table 9). We then made similar comparisons between the regression estimates of the following pairs, respectively: the low-collectivistic group (US) and the high-collectivistic groups (Greece and India). 
Insert Table 9 about here

The equality of the structural coefficients test showed no significant difference between the sets of regression estimates for the two high collectivistic groups. However, the coefficients of the low collectivistic group (US) were significantly different to both Greece and India, respectively. The results suggested that the data of individuals from high- and lowcollectivistic cultures did not demonstrate the same fit patterns, while the results from the two high-collectivistic groups were essentially very similar. Thus, culture seems to serve as a moderator between relationship-specific avoidant attachment and just-treatment expectations, if the new leader is similar to the previous one.

Hence, those individuals in the US who have a highly avoidant attachment relationship to their current leader are more likely to be influenced by their previous leader: Specifically, they are more likely to transfer previous just-treatment evaluations to a new similar leader.

We also found a positive relationship between relationship-specific fearful attachment and just-treatment expectations at T2 in both the Greek (Table 8, Model 3; $b=.30, S E=.12$, $p=.02$ ) and the Indian samples (Model 3; $b=.28, S E=.15, p<.059)$. We again examined whether the partial coefficients of the fit measures differed across groups. The test on the equality of structural coefficients across groups (see Table 10) again showed that there was no significant difference between the sets of regression estimates for the Greek and the Indian culture.

Insert Table 10 about here 
The coefficients of the low-collectivistic group (US) were significantly different to both the high-collectivistic groups (Greece and India, respectively), which indicates that in the higher similarity leader condition fearful individuals in Greece or in India are more likely to expect higher just-treatment behavior, compared to the American sample.

Perceived Leader Effectiveness. The US sample was compared to samples in Greece and in India with regard to perceived leader effectiveness. Again, examining seemingly unrelated regressions, we combined the estimates from the three equations (of the three samples) in one vector for the parameters with a robust variance matrix. Examining whether partial coefficients of fit measures differed across the groups by simultaneously testing the difference in regression coefficients, revealed that in the higher similarity leader condition, only the American sample showed significant effects with regard to global avoidant attachment. Interestingly, in the US sample, there was an initial negative relationship between global avoidance and leader effectiveness at T2 (Table 11, United States; $b=-.56, S D=.14$, $p<.001)$

Insert Table 11 about here

However, the higher similarity leader condition moderated this association and rendered it positive (Table 11, US; $b=.64 ; S D=.19, p=.001$ ). These results are directly opposite to those found in the original Greek sample in the similar-leader condition. No significant results were found in the Indian sample.

\section{Brief Discussion}

The analyses presented here suggest that culture can be a potent moderating factor in relationships between attachment orientations and the transfer of leader expectations. As in Study 1, experimentally induced leader similarity activated insecure attachment orientations 
in a collectivistic (India) and in an individualistic culture (the US), yet in different ways. In India, relationship-specific avoidance was negatively associated with and relationshipspecific fearfulness (high avoidance and high anxiety) was positively associated with just treatment expectations of a new similar leader as anticipated by $\mathrm{H} 2$. These results, were similar in direction, did not differ between the two collectivistic countries, were significantly different from results observed in the individualistic culture (US) and in line with our initial expectations regarding cultural variability: negative new similar leader expectations associated with avoidance and positive expectations associated with anxiety will be weakened or become non-significant in an individualistic culture. In addition, when activated by new leader similarity to the previous leader, in the individualistic culture, global avoidance was positively associated with new similar leader effectiveness perceptions. Taken together, these findings suggest that the cultural context can shape the relative emphasis activated attachment orientations place on self and significant others, and likely influences respective leader perceptions.

\section{General Discussion}

Previous experiences with and mental representations of significant others constitute an important blueprint that allows people to make sense of and evaluate future leaders (Ritter \& Lord, 2007). We examined attachment orientations as possible predictors of the transfer of such leader expectations, in particular, just-treatment expectations and perceived leader effectiveness, in cultures that differ in terms of their central cultural inclination towards individualism versus collectivism.

Overall, an online idiographic manipulation of leader similarity was successful in activating attachment orientations and led to a shift in leader behavioral expectations. In Study 1 (Greece), the invoked similarity between participants' current and the presented highly similar new leader likely triggered positive models of significant other inherent in 
relationship-specific anxious attachment working models $(H 2 b)$. Effectiveness evaluations of the new leader were also positively associated with global anxious attachment. Individuals with a global anxious attachment orientation attributed higher effectiveness scores to a new, highly similar leader than individuals who scored lower on this dimension. Therefore, in line with $H 3 a$ and $H 3 b$, relationship-specific anxious attachment predicts more contextual-related just treatment expectations, just as global anxious attachment predicts leader effectiveness expectations.

We found a reverse pattern of relationships for avoidant attachment and new leader expectations in line with $H 2 b$. Relationship-specific and global avoidant orientations were associated, respectively, with low just treatment and effectiveness expectations of the new, highly similar leader. Also, individuals who scored high on global avoidant attachment had the lowest just treatment expectations in the high leader similarity condition, compared to all other attachment orientations. Notably, the observed results remained significant when controlling for trait anxiety, relationship importance, daily contact with the leader, and the Big Five. This suggests that the transfer process was attributable to the defensive processes associated with insecure attachment orientations.

These findings lend support to social-cognitive explanations on the links between projective and perceptual processes associated with insecure individuals' attributions to others (Mikulincer \& Horesh, 1999) and extend those explanations to leader perception. The here presented studies suggest that high leader similarity is a key trigger of significant other attachment cognitions (Baldwin \& Meunier, 1999). Hence, attachment cognitions are not only activated within close relationships, but the attachment schema can cover leaderfollower expectations and perceptions in line with evidence for the pervasive character of attachment working models (Turan, 2016). The findings from the present studies extend research on the transfer of relationship expectations (Brumbaugh \& Fraley, 2006, 2007) to 
the leadership field. In line with previous research (Brumbaugh \& Fraley, 2006; Overall et al., 2003) a more emotional relationship context (ratings of just treatment expectations) was associated with the activation of relationship-specific working models.

Importantly, patterns of transfer of new leader expectations associated with avoidant attachment orientations differed as a function of cultural context. There has been increasing evidence that culture can moderate attachment-related relational processes (Friedman et al., 2010), including leadership perceptions of avoidant leaders (Kafetsios et al., 2014).

As outlined, anxious attachment is more prevalent in collectivistic cultures, likely because by being highly interdependent, collectivistic cultures emphasize other-orientation. Our research confirmed this pattern, as anxious attachment scores obtained in India were highest compared to Greece or the US (Study 2). In collectivistic cultures, anxious followers' typical other-oriented schemas can be reinforced by the collectivistic culture's inclination for other-orientation in social relationships. In such cultures, conversely, avoidant persons are typically asked to be more socially engaging and other-oriented, which is not in their nature (Friedman et al., 2010). Priming similarity in leader-traits, coupled with the culture's otheroriented cultural mandate is not in line with avoidant followers' tendencies to distance themselves from others and focus on the self (Mikulincer et al., 1998). Accordingly, the present studies found that avoidant individuals in Greece and in India, two collectivistic cultures, held low just treatment expectations of a new, similar leader. These findings are in stark contrast to avoidant individuals in the US, an individualistic culture, who tend to hold high just-treatment expectations of new, highly similar leaders. Hence, similar leaders were evaluated more positively by avoidant persons in the US, which is atypical of avoidant individuals. This is an important finding, as it suggests that overarching cultural schemas (a higher focus on the self in individualistic cultures) may be associated with a shift in avoidant persons' negative transference to a new similar leader. This change is likely because at the 
point of activation the self-other balance shifts from the self to the new 'significant other'. It is likely that avoidant persons' high self-focus and self-determination in individualist cultures, due to the fit of personal to cultural schemas (Fulmer et al., 2010), may dampen and even reverse avoidant persons' defenses towards new leaders. Such an explanation of how culture may moderate avoidant individuals' leader expectations is in line with socialcognitive models of personality built on different self-significant other knowledge structures (Andersen \& Chen, 2002). The present studies further strengthen that view by that culture's stronger association with the avoidant attachment orientation, which is considered to have more cognitive defenses (Gillath, Giesbrecht, \& Shaver, 2009). However, in our studies, this point only seems to hold true for behavioral expectations, such as expectations of just treatment. Results did not apply to more cognitive evaluations of leader-effectiveness. Indeed, a model including all three cultures did not reveal any significant results.

\section{Limitations and Future Research}

The present studies are not devoid of limitations. First, we relied on Andersen's transference measurement method, as adopted by Ritter \& Lord (2007). We slightly adapted this method using a pre-determined set of attributes, instead of asking participants to retrieve attributes from memory. One could argue that his list of provided attributes may not truly reflect participant leader perceptions. Nonetheless, we repeatedly replicated previous results, which gives us confidence in this method's suitability and reliability. In addition, the method greatly simplifies execution and allows for quicker data collection and much larger samples than manual execution allows.

A second potential limitation was the use of online participant pools for two of the three samples. Yet, the MTurk population is not very different from traditional participants (Paolacci \& Chandler, 2014). Although, we do acknowledge the lack of controls regarding experimental conditions with online populations, Study 1 participants, although not recruited 
via MTurk, also filled out the survey online at their own discretion. Therefore, the conditions were very similar across all three samples.

Third, the present studies measured the transference of persons' judgments and evaluations regarding a new leader and not the transference of knowledge structures per se (i.e., Ritter \& Lord, 2007). The activation of specific preferences and evaluations in the higher similarity leader condition could reflect both bottom-up processes of transference as suggested in this paper, and also top-down processes of leader categorization (Lord, Foti, \& De Vader, 1984). Hence, it is possible that anxious and avoidant attachment schemas activate specific (and different) hierarchically arranged implicit leadership schemas, as a result of stored categorization of how new leaders should be like. Although plausible, this explanation cannot fully account as to why such schemas are not at all present in the low similarity leader condition or why these schemas are so volatile in different cultures.

In addition, we did not account for relationship quality as a possible moderating factor in the transference process. If individuals find themselves in low-quality relationships with their leader, this might impact the transference process itself. However, although we did not directly test for such a possible moderation, available proxy variables, namely positive and negative affect in the pre-test, relationship importance and daily contact in the subsequent studies, suggested that the transference process was impervious to those indicators. This is also in line with Brumbaugh and Fraley (2006).

Finally, the use of a categorical cultural factor forms another limitation, as we accounted for cultural differences by testing our hypotheses in three different populations and focusing on independence-interdependence. We encourage scholars to adopt individual-level measures of cultural dimensions. Doing so would allow researchers to disentangle which specific cultural aspects most strongly influence the relationship between attachment orientations and the transference of behavioral expectations. 
RUNNING HEAD: Attachment in leader transfer

\section{Conclusion}

Limitations notwithstanding, the findings from this research suggest that attachment orientations are potent antecedents to social-cognitive processes related to the transfer of expectations from a previous to a new leader. Global and relationship-specific attachment working models play a role, depending on whether the dependent variable describes broader cognitive (e.g., perceived leader effectiveness), or more episodic-related emotional concepts (e.g., expectations of just treatment). These observations are in line with a dynamic view of adult attachment organization (Mikulincer \& Shaver, 2007) and with broader models of situated social cognition (e.g., Smith \& Semin, 2007) applied to implicit leadership theories. 


\section{References}

Agishtein, P., \& Brumbaugh, C. (2013). Cultural variation in adult attachment: The impact of ethnicity, collectivism, and country of origin. Journal of Social, Evolutionary, and Cultural Psychology, 7(4), 384.

Andersen, S. M., \& Chen, S. (2002). The relational self: an interpersonal social-cognitive theory. Psychological Review, 109(4), 619.

Andersen, S. M., \& Glassman, N. S. (1996). Responding to significant others when they are not there: Effects on interpersonal inference, motivation, and affect. In R. Sorrentino \& E. T. Higgins (Eds.), Handbook of motivation and cognition (Vol. 3, pp. 272-331). New York: Guilford Press.

Anderson, N. H. (1968). Likableness ratings of 555 personality-trait words. Journal of Personality and Social Psychology, 9(3), 272.

Baldwin, M. W., Keelan, J. P. R., Fehr, B., Enns, V., \& Koh-Rangarajoo, E. (1996). Socialcognitive conceptualization of attachment working models: Availability and accessibility effects. Journal of Personality and Social Psychology, 71(1), 94.

Bowlby, J. (1969). Attachment and loss, volume $i$ : Attachment. London: Random House.

Brennan, K.A., Clark, C.L., \& Shaver, P.R. (1998). Self-report measurement of adult attachment: An integrative overview. In J.A. Simpson \& W.S. Rholes (Eds.), Attachment Theory and Close Relationships (pp. 46-76). New York: Guilford Press.

Brewer, M. B., \& Gardner, W. (1996). Who is this" We"? Levels of collective identity and self representations. Journal of Personality and Social Psychology, 71(1), 83.

Brislin, R. W. (1970). Back-translation for cross-cultural research. Journal of Cross-Cultural Psychology, 1(3), 185-216.

Brumbaugh, C. C., \& Fraley, C. (2006). Transference and attachment: How do attachment patterns get carried forward from one relationship to the next? Personality and Social Psychology Bulletin, 32(4), 552-560.

Brumbaugh, C. C., \& Fraley, C. (2007). Transference of attachment patterns: How important relationships influence feelings toward novel people. Personal Relationships, 14(4), 513-530.

Cheng, S. T., \& Kwan, K. W. (2008). Attachment dimensions and contingencies of selfworth: The moderating role of culture. Personality and Individual Differences, 45(6), 509-514.

Collins, N. \& Read, S. (1994). Representations of attachment: The structure and function of working models. In K. Bartholomew and D. Perlman (Eds.), Advances in Personal Relationships: Attachment process in adulthood (Vol 5 pp. 53-90). London: Jessica Kingsley.

Colquitt, J. A. (2001). On the dimensionality of organizational justice: A construct validation of a measure. Journal of Applied Psychology, 86(3), 386-400.

Colquitt, J. A., \& Rodell, J. B. (2011). Justice, trust, and trustworthiness: A longitudinal analysis integrating three theoretical perspectives. Academy of Management Journal, 54(6), 1183-1206.

Davidovitz, R., Mikulincer, M., Shaver, P. R., Izsak, R., \& Popper, M. (2007). Leaders as attachment figures: Leaders' attachment orientations predict leadership-related mental representations and followers' performance and mental health. Journal of Personality and Social Psychology, 93, 632-650. 
Faul, F., Erdfelder, E., Lang, A.-G., \& Buchner, A. (2007). G* Power 3: A flexible statistical power analysis program for the social, behavioral, and biomedical sciences. Behavior research methods, 39(2), 175-191.

Fitzsimons, G. M., \& Bargh, J. A. (2003). Thinking of you: nonconscious pursuit of interpersonal goals associated with relationship partners. Journal of Personality and Social Psychology, 84(1), 148.

Fraley, C., \& Brumbaugh, C. C. (2004). A dynamical systems approach to conceptualizing and studying stability and change in attachment security. Adult attachment: Theory, research, and clinical implications, 86-132.

Fraley, C., Heffernan, M. E., Vicary, A. M., \& Brumbaugh, C. C. (2011). The experiences in close relationships-Relationship Structures Questionnaire: A method for assessing attachment orientations across relationships. Psychological Assessment, 23(3), 615.

Fraley, R. C., Hudson, N. W., Heffernan, M. E., \& Segal, N. (2015). Are adult attachment styles categorical or dimensional? A taxometric analysis of general and relationshipspecific attachment orientations. Journal of Personality and Social Psychology, $109(2), 354$.

Friedman, M., Rholes, W. S., Simpson, J., Bond, M., Diaz-Loving, R., \& Chan, C. (2010). Attachment avoidance and the cultural fit hypothesis: A cross-cultural investigation. Personal Relationships, 17(1), 107-126.

Fulmer, C. A., Gelfand, M. J., Kruglanski, A. W., Kim-Prieto, C., Diener, E., Pierro, A., \& Higgins, E. T. (2010). On "feeling right" in cultural contexts: How person-culture match affects self-esteem and subjective well-being. Psychological Science, 21(11), 1563-1569.

Gillath, O., Giesbrecht, B., \& Shaver, P. R. (2009). Attachment, attention, and cognitive control: Attachment style and performance on general attention tasks. Journal of Experimental Social Psychology, 45(4), 647-654.

Goldberg, L. R., Johnson, J. A., Eber, H. W., Hogan, R., Ashton, M. C., Cloninger, C. R., \& Gough, H. G. (2006). The international personality item pool and the future of publicdomain personality measures. Journal of Research in Personality, 40(1), 84-96.

Hinkley, K., \& Andersen, S. M. (1996). The working self-concept in transference: Significant-other activation and self change. Journal of Personality and Social Psychology, 71(6), 1279.

Hofstede, G. H. (2001). Culture's consequences: Comparing values, behaviors, institutions and organizations across nations. Thousand Oaks, CA Sage.

Jarymowicz, M. (1992). Self, we, and other(s): Schemata, distinctiveness, and altruism. In P. M. Oliner, S. P. Oliner, L. Baron, L. A. Blum, D. L. Krebs, \& M. Z. Smolenska (Eds.), Embracing the other: Philosophical, psychological, and historical perspectives on altruism (pp. 194-212). New York: New York University Press

Kafetsios, K., Athanasiadou, M., \& Dimou, N. (2014). Leaders' and subordinates' attachment orientation, emotion regulation and affect at work: A multilevel analysis. The Leadership Quarterly, 25, 3, 512-527.

Klohnen, E. C., Weller, J. A., Luo, S., \& Choe, M. (2005). Organization and predictive power of general and relationship-specific attachment models: One for all, and all for one? Personality and Social Psychology Bulletin, 31(12), 1665-1682.

Lord, R. G., \& Brown, D. J. (2003). Leadership processes and follower self-identity: Psychology Press.

Lord, R. G., Diefendorff, J. M., Schmidt, A. M., \& Hall, R. J. (2010). Self-regulation at work. Annual Review of Psychology, 61, 543-568. 
Lord, R. G., Foti, R. J., \& De Vader, C. L. (1984). A test of leadership categorization theory: Internal structure, information processing, and leadership perceptions. Organizational Behavior and Human Performance, 34(3), 343-378.

Mason, W., \& Suri, S. (2012). Conducting behavioral research on Amazon's Mechanical Turk. Behavior Research Methods, 44(1), 1-23.

Mayseless, O., \& Popper, M. (2018). Attachment and leadership: review and new insights. Current Opinion in Psychology, 25, 157.Mikulincer, M., \& Horesh, N. (1999). Adult attachment style and the perception of others: the role of projective mechanisms. Journal of Personality and Social Psychology, 76(6), 1022.

Mikulincer, M., \& Nachshon, O. (1991). Attachment styles and patterns of self-disclosure. Journal of Personality and Social Psychology, 61(2), 321.

Mikulincer, M., Orbach, I., \& Iavnieli, D. (1998). Adult attachment style and affect regulation: Strategic variations in subjective self-other similarity. Journal of Personality and Social Psychology, 75(2), 436.

Mikulincer, M., \& Shaver, P. R. (2007). Attachment in adulthood: Structure, dynamics, and change. New York: Guilford Press.

Overall, N. C., Fletcher, G. J., \& Friesen, M. D. (2003). Mapping the intimate relationship mind: Comparisons between three models of attachment representations. Personality and Social Psychology Bulletin, 29(12), 1479-1493.

Paolacci, G., \& Chandler, J. (2014). Inside the turk understanding mechanical turk as a participant pool. Current Directions in Psychological Science, 23(3), 184-188.

Pierce, T., \& Lydon, J. E. (2001). Global and specific relational models in the experience of social interactions. Journal of Personality and Social Psychology, 80(4), 613.

Pierro, A., \& Kruglanski, A. W. (2008). "Seizing and freezing" on a significant-person schema: need for closure and the transference effect in social judgment. Personality and Social Psychology Bulletin, 34(11), 1492-1503.

Pietromonaco, P. R., \& Barrett, L. F. (2000). The internal working models concept: What do we really know about the self in relation to others?. Review of General Psychology, $4(2), 155-175$.

Popper, M. (2004). Leadership as a relationship. Journal for the Theory of Social Behavior, $34,107-125$.

Richards, D. A., \& Schat, A. C. (2011). Attachment at (not to) work: applying attachment theory to explain individual behavior in organizations. Journal of Applied Psychology, 96(1), 169-182.

Ritter, B. A., \& Lord, R. G. (2007). The impact of previous leaders on the evaluation of new leaders: an alternative to prototype matching. Journal of Applied Psychology, 92(6), 1683.

Schmitt, D. P., Alcalay, L., Allensworth, M., Allik, J., Ault, L., Austers, I., .. C Cunen, M. A. B. (2004). Patterns and universals of adult romantic attachment across 62 cultural regions are models of self and of other pancultural constructs? Journal of CrossCultural Psychology, 35(4), 367-402.

Smith, E. R., \& Semin, G. R. (2007). Situated social cognition. Current Directions in Psychological Science, 16(3), 132-135.

Spielberger, C.D. (1983).State-trait anxiety inventory for adults. Palo Alto, CA: Mind GardenStataCorp. (2011). Stata Statistical Software: Release 12. College Station, TX: StataCorp LP.

Thomas, G., Martin, R., Epitropaki, O., Guillaume, Y., \& Lee, A. (2013). Social cognition in leader-follower relationships: Applying insights from relationship science to understanding relationship-based approaches to leadership. Journal of Organizational Behavior, 34(S1), S63-S81. 
Turan, B. (2016). Processing information about support exchanges in close relationships: The role of a knowledge structure. Frontiers in psychology, 7, 259.

van IJzendnorn, M. H., \& Sagi, A. (1999). Cross-cultural patterns of attachment: Universal and contextual dimensions. In J. Cassidy \& P. R. Shaver (Eds.), Handbook of attachment: Theory, research, and clinical applications (pp. 713-734). New York: Guilford Press.

Watson, D., \& Clark, L. A. (1991). Self-versus peer ratings of specific emotional traits: Evidence of convergent and discriminant validity. Journal of Personality and Social Psychology, 60(6), 927.

Zhao, X., et al. (2018). Eighty years after Lewin: The role of collectivism and attachment style in friendship networks across 21 nations. MS under review. 
RUNNING HEAD: Attachment in leader transfer

Table 1

Correlations of Studied Variables (Pre-Test)

\begin{tabular}{|c|c|c|c|c|c|c|c|c|c|c|c|c|}
\hline & & M & $\mathrm{SD}$ & 1 & 2 & 3 & 4 & 5 & 6 & 7 & 8 & 9 \\
\hline 1 & Leader similarity condition & .49 & .50 & & & & & & & & & \\
\hline 2 & Just treatment $-\mathrm{T} 1$ & 3.84 & 1.07 & -.05 & $(.93)$ & & & & & & & \\
\hline 3 & Perceived leader effectiveness $-\mathrm{T} 1$ & 3.49 & 1.19 & -.05 & $.76^{* * *}$ & - & & & & & & \\
\hline 4 & Positive affect $-\mathrm{T} 1$ & 2.96 & .92 & -.02 & $.20^{* *}$ & $.22^{* *}$ & $(.92)$ & & & & & \\
\hline 5 & Negative affect $-\mathrm{T} 1$ & 1.30 & .61 & -.08 & -.11 & -.06 & $-.15^{*}$ & $(.96)$ & & & & \\
\hline 6 & Just treatment $-\mathrm{T} 2$ & 3.29 & 1.08 & .11 & $.30^{* * *}$ & $.22^{* *}$ & -.08 & -.03 & $(.94)$ & & & \\
\hline 7 & Perceived leader effectiveness - T2 & 2.40 & 1.03 & .08 & $.22^{* *}$ & $.26^{* * *}$ & .14 & -.01 & $.51^{* * *}$ & & & \\
\hline 8 & Positive affect $-\mathrm{T} 2$ & 2.82 & .92 & .07 & $.21^{* *}$ & $.23^{* * *}$ & $.81^{* * *}$ & -.15 & .09 & $.23^{* * *}$ & $(.92)$ & \\
\hline 9 & Negative affect $-\mathrm{T} 2$ & 1.40 & .71 & -.08 & .02 & -.02 & -.02 & $.50^{* * *}$ & $-.17^{*}$ & .05 & -.13 & $(.94)$ \\
\hline
\end{tabular}

Leader similarity condition: low similarity to previous leader $=0$; higher similarity to previous leader $=1$; reliability alphas in parentheses on the diagonal where appropriate (perceived leader effectiveness is a one-item measure); ${ }^{* * *} p<.001 ;{ }^{* *} p<.01 ;{ }^{*} p<.05 ; n=211$. 
RUNNING HEAD: Attachment in leader transfer

Table 2

Correlations of Studied Variables (Study 1 - Greek workers)

\begin{tabular}{|c|c|c|c|c|c|c|c|c|c|c|c|c|c|c|c|c|c|c|c|c|c|}
\hline & & $\mathrm{M}$ & SD & 1 & 2 & 3 & 4 & 5 & 6 & 7 & 8 & 9 & 10 & 11 & 12 & 13 & 14 & 15 & 16 & 17 & 18 \\
\hline 1 & LSC & .46 & .50 & - & & & & & & & & & & & & & & & & & \\
\hline 2 & Leader effectiveness - T2 & 3.22 & .88 & .13 & - & & & & & & & & & & & & & & & & \\
\hline 3 & Leader effectiveness - T1 & 3.77 & 1.1 & .10 & .04 & - & & & & & & & & & & & & & & & \\
\hline 4 & Just-treatment expectation - $\mathrm{T} 2$ & 3.47 & .84 & -.13 & $.35^{* * *}$ & .06 & (.87) & & & & & & & & & & & & & & \\
\hline 5 & Just treatment $-\mathrm{T} 1$ & 3.79 & 1.01 & .04 & -.11 & $.59^{* * *}$ & .00 & $(.91)$ & & & & & & & & & & & & & \\
\hline 6 & Anxiety (GL) & 3.35 & 1.21 & -.07 & $.28^{* *}$ & -.16 & .10 & $-.23^{*}$ & $(.94)$ & & & & & & & & & & & & \\
\hline 7 & Avoidance (GL) & 3.45 & .93 & -.01 & .18 & .02 & -.06 & -.07 & $.48^{* * *}$ & $(.90)$ & & & & & & & & & & & \\
\hline 8 & Anxiety (GL) * Avoidance (GL) & 12.09 & 6.41 & -.02 & -.10 & .08 & -.13 & .08 & $-.42^{* * *}$ & $.49^{* * *}$ & - & & & & & & & & & & \\
\hline 9 & Anxiety (RS) & 3.12 & 1.38 & -.16 & .03 & $-.36^{* * *}$ & .15 & $-.53^{* * *}$ & $.36^{* * *}$ & .08 & -.16 & $(.80)$ & & & & & & & & & \\
\hline 10 & Avoidance (RS) & -3.89 & 1.53 & $-.21^{*}$ & .09 & $-.67^{* * *}$ & .02 & $-.56^{* * *}$ & $.38^{* * *}$ & $.20^{\dagger}$ & -.06 & .47 & $(.93)$ & & & & & & & & \\
\hline 11 & Anxiety (RS)*Avoidance (RS) & 12.09 & 6.41 & -.16 & .16 & $-.54^{* * *}$ & .04 & $-.44^{* * *}$ & $.24^{* * *}$ & $.20^{*}$ & .04 & .12 & $.81^{* * *}$ & - & & & & & & & \\
\hline 12 & Openness to experiences & 3.80 & .73 & .10 & -.13 & .04 & -.07 & .03 & $-.37^{* * *}$ & $-.37^{* * *}$ & -.01 & -.01 & -.13 & -.11 & $(.68)$ & & & & & & \\
\hline 13 & Conscientiousness & 3.95 & .75 & .00 & $-.29^{* *}$ & .07 & -.14 & .17 & $-.38^{* * *}$ & .01 & $.33^{* *}$ & -.14 & -.12 & -.08 & .12 & $(.60)$ & & & & & \\
\hline 14 & Extraversion & 3.26 & .92 & .04 & .04 & .08 & .12 & .00 & $-.20^{*}$ & $-.44^{* * *}$ & $-.26^{* *}$ & -.07 & $-.21^{*}$ & -.16 & $.35^{* * *}$ & .05 & $(.74)$ & & & & \\
\hline 15 & Agreeableness & 3.96 & .68 & .07 & -.10 & .10 & -.06 & .03 & $-.33^{* * *}$ & $-.53^{* * *}$ & $-.28^{* * *}$ & -.04 & $-.22^{*}$ & $-.24^{*}$ & $.50^{* * *}$ & .08 & $.46^{* * *}$ & $(.74)$ & & & \\
\hline 16 & Neuroticism & 3.19 & .83 & -.10 & .16 & -.18 & -.04 & -.03 & $.57^{* * *}$ & $.28^{* * *}$ & $-.30^{* *}$ & $.20^{\dagger}$ & $.28^{* *}$ & $.23^{*}$ & $-.36^{* * *}$ & $-.31^{*}$ & -.12 & $-.25^{* *}$ & $(.66)$ & & \\
\hline 17 & Trait anxiety & 2.17 & .55 & -.04 & .16 & -.14 & -.07 & -.12 & $.68^{* * *}$ & $.48^{* * *}$ & -.11 & $.23^{*}$ & $.35^{* * *}$ & $.22^{*}$ & $-.34^{* * *}$ & $-.29^{*}$ & $-.33^{* * *}$ & $-.35^{* * *}$ & $.68^{* * *}$ & $(.87)$ & \\
\hline 18 & Relationship importance & 3.42 & 1.17 & .12 & -.01 & $.72^{* * *}$ & .11 & $.61^{* * *}$ & $-.27^{* *}$ & -.02 & .14 & $-.37^{* * *}$ & $-.71^{* * *}$ & $-.56^{* * *}$ & .01 & .17 & .11 & .01 & -.21 & $-.28^{* *}$ & - \\
\hline 19 & Daily contact & 3.55 & 1.18 & .07 & .06 & $.35^{* * *}$ & $.20^{*}$ & $.17^{*}$ & -.12 & -.06 & .10 & $-.27^{* *}$ & $-.29^{* *}$ & $-.21^{*}$ & $\begin{array}{r}-.19 \\
\end{array}$ & .01 & .08 & $\begin{array}{r}-.02 \\
-.02\end{array}$ & -.07 & -.08 & $.41^{* * *}$ \\
\hline
\end{tabular}

LSC = Leader similarity condition (low similarity to previous leader = 0 ; higher similarity to previous leader $=1$ ); GL = Global attachment; RS = Relationship-specific attachment at $\mathrm{T} 1 ;{ }^{* * *} p<.001 ;{ }^{* *} p<.01 ;{ }^{*} p<.05 ; n=95$. 
RUNNING HEAD: Attachment in leader transfer

Table 3

Regression Estimates (Greece): Predicting Expectations of Just Treatment T2

\begin{tabular}{|c|c|c|c|c|c|}
\hline \multirow{2}{*}{$\begin{array}{l}\text { Variable } \\
\text { LSC }\end{array}$} & \multirow{2}{*}{$\begin{array}{c}\text { Model 1 } \\
-2.63^{* * *}\end{array}$} & \multirow{2}{*}{$\frac{\text { Model 2 }}{-3.29^{* *}}$} & \multirow{2}{*}{$\frac{\text { Model 3 }}{-3.11^{* *}}$} & \multicolumn{2}{|c|}{ 95\% Confidence Interval } \\
\hline & & & & -4.98 & -1.24 \\
\hline & $(-4.43)$ & $(-3.61)$ & $(-3.31)$ & & \\
\hline \multirow[t]{2}{*}{ Just treatment $\mathrm{T} 1$} & $-.25^{*}$ & -.17 & -.15 & -.44 & .14 \\
\hline & $(-2.54)$ & $(-1.23)$ & $(-1.08)$ & & \\
\hline \multirow[t]{2}{*}{ LSC *Just treatment $-\mathrm{T} 1$} & $.63^{* * *}$ & $.58^{* * *}$ & $.54^{* *}$ & .16 & .91 \\
\hline & $(4.28)$ & (3.19) & $(2.84)$ & & \\
\hline \multirow[t]{2}{*}{ Anxiety (RS) } & & -.05 & -.09 & -.10 & .28 \\
\hline & & $(.58)$ & $(.93)$ & & \\
\hline \multirow[t]{2}{*}{ LSC * Anxiety (RS) } & & .23 & $.23^{*}$ & .00 & .46 \\
\hline & & $(1.91)$ & $(2.00)$ & & \\
\hline \multirow[t]{2}{*}{ Avoidance (RS) } & & .23 & .29 & -.18 & . 77 \\
\hline & & $(.93)$ & $(1.22)$ & & \\
\hline \multirow[t]{2}{*}{ LSC * Avoidance (RS) } & & $-.58^{*}$ & $-.57^{*}$ & -1.10 & -.05 \\
\hline & & $(-2.12)$ & $(-2.16)$ & & \\
\hline \multirow[t]{2}{*}{ Anxiety (RS) * Avoidance (RS) } & & -.07 & -.06 & -.19 & .07 \\
\hline & & $(-.94)$ & $(-0.91)$ & & \\
\hline \multirow[t]{2}{*}{ LSC *Anxiety $(\mathrm{RS}) *$ Avoidance $(\mathrm{RS})$} & & $.18^{*}$ & $.18^{*}$ & .02 & .34 \\
\hline & & $(2.18)$ & $(2.22)$ & & \\
\hline \multirow[t]{2}{*}{ Constant } & & $4.11^{* * *}$ & $3.24^{* * *}$ & 1.59 & 4.9 \\
\hline & & $(5.79)$ & $(3.89)$ & & \\
\hline $\mathrm{F}$ & 6.55 & 4.15 & 4.07 & & \\
\hline $\mathrm{R}^{2}$ & $.15^{* * *}$ & $.23^{* * *}$ & $.29^{* * *}$ & & \\
\hline
\end{tabular}

LSC $=$ Leader similarity condition (low similarity to previous leader $=0$; higher similarity to previous leader $=$ 1); RS = Relationship-specific attachment at Time 1; controlling for trait anxiety, relationship importance and daily contact in Model 3; (Big-Five personality dimensions did not change results, and are available from the authors); Confidence Intervals correspond to Model 3; robust $t$-statistics in parentheses; unstandardized coefficients; ${ }^{* * *} p<.001 ;{ }^{* *} p<.01 ;{ }^{*} p<.05 ; n=95$. 
RUNNING HEAD: Attachment in leader transfer

Table 4

Regression Estimates (Greece): Predicting Perceived Leader Effectiveness T2

\begin{tabular}{|c|c|c|c|c|c|}
\hline Variable & Model 1 & Model 2 & Model 3 & \multicolumn{2}{|c|}{ 95\% Confidence Interval } \\
\hline LSC & $\begin{array}{c}-1.90 \\
(-3.02)\end{array}$ & $\begin{array}{l}-3.66^{* *} \\
(-2.85)\end{array}$ & $\begin{array}{l}-3.91^{* *} \\
(-2.94)\end{array}$ & -6.56 & -1.26 \\
\hline Perceived leader effectiveness $\mathrm{T} 1$ & $\begin{array}{c}-.23 \\
(-1.99)\end{array}$ & $\begin{array}{c}-.22 \\
(-1.91)\end{array}$ & $\begin{array}{c}-.16 \\
(-1.40)\end{array}$ & -.39 & .07 \\
\hline LSC *Perceived leader effectiveness T1 & $\begin{array}{c}.56 \\
(3.40)\end{array}$ & $\begin{array}{l}.60^{* * *} \\
(3.76)\end{array}$ & $\begin{array}{l}.61^{* * *} \\
(3.71)\end{array}$ & .28 & .93 \\
\hline Anxiety (GL) & & $\begin{array}{c}-.04 \\
(-.18)\end{array}$ & $\begin{array}{c}-.08 \\
(-.36)\end{array}$ & -.51 & .35 \\
\hline LSC * Anxiety (GL) & & $\begin{array}{c}.65 \\
(1.96)\end{array}$ & $\begin{array}{c}.72^{*} \\
(2.09)\end{array}$ & .04 & 1.41 \\
\hline Avoidance (GL) & & $\begin{array}{c}.42 \\
(1.11)\end{array}$ & $\begin{array}{c}.52 \\
(1.32)\end{array}$ & -.26 & 1.30 \\
\hline LSC * Avoidance (GL) & & $\begin{array}{c}-.88^{*} \\
(-1.89)\end{array}$ & $\begin{array}{l}-1.00^{*} \\
(-2.05)\end{array}$ & -1.97 & -.03 \\
\hline Anxiety (GL) * Avoidance (GL) & & $\begin{array}{c}-.17 \\
(-1.40)\end{array}$ & $\begin{array}{c}-.20^{\dagger} \\
(-1.66)\end{array}$ & -.43 & .04 \\
\hline LSC * Anxiety (GL) * Avoidance (GL) & & $\begin{array}{c}.40^{*} \\
(2.42)\end{array}$ & $\begin{array}{c}.44^{*} \\
(2.62)\end{array}$ & .10 & .77 \\
\hline Constant & $\begin{array}{c}3.95^{* * *} \\
(10.52)\end{array}$ & $\begin{array}{c}4.12^{* * *} \\
(4.30)\end{array}$ & $\begin{array}{l}4.23^{* * *} \\
(4.34)\end{array}$ & 2.29 & 6.18 \\
\hline $\begin{array}{l}\mathrm{F} \\
\mathrm{R}^{2}\end{array}$ & $\begin{array}{l}4.57 \\
.14^{* *}\end{array}$ & $\begin{array}{l}3.50 \\
.27^{* * *}\end{array}$ & $\begin{array}{l}2.88 \\
.28^{* *}\end{array}$ & & \\
\hline
\end{tabular}

LSC $=$ Leader similarity condition (low similarity to previous leader $=0$; higher similarity to previous leader $=$

1); GL = Global attachment; controlling for trait anxiety, relationship importance and daily contact in Model 3;

(Big-Five personality dimensions did not change results, and are available from the authors); Confidence

Intervals correspond to Model 3; robust $t$-statistics in parentheses; unstandardized coefficients; ${ }^{* * *} p<.001 ;{ }^{* *} p<$

$.01 ;{ }^{*} p<.05 ; n=95$. 
RUNNING HEAD: Attachment in leader transfer

Table 5

Means and Standard Deviations by Culture for Primary Variables

\begin{tabular}{l|ccccccccccc}
\hline & \multicolumn{2}{c}{ United States } & \multicolumn{3}{c}{ Greece } & \multicolumn{4}{c}{ India } \\
& $M$ & $S D$ & $N$ & $M$ & $S D$ & $N$ & $M$ & $S D$ & $N$ \\
\hline Anxiety (GL) & 2.87 & 1.32 & 139 & 3.35 & 1.21 & 95 & 3.68 & 1.19 & 109 \\
Avoidance (GL) & 3.93 & 1.46 & 139 & 3.45 & .93 & 95 & 3.36 & .86 & 109 \\
Anxiety (RS) & 2.35 & 1.26 & 139 & 3.12 & 1.38 & 95 & 3.44 & 1.61 & 109 \\
Avoidance (RS) & 3.93 & 1.75 & 139 & 3.89 & 1.53 & 95 & 3.42 & 1.15 & 109 \\
Just-treatment expectations - T2 & 3.31 & 1.08 & 137 & 3.47 & .84 & 95 & 3.46 & .76 & 109 \\
Perceived leader effectiveness - T2 & 2.36 & 1.12 & 137 & 3.22 & .88 & 95 & 3.49 & 1.08 & 109 \\
\hline
\end{tabular}

$\mathrm{GL}=$ Global attachment; RS = Relationship-specific attachment; unstandardized coefficients. 
RUNNING HEAD: Attachment in leader transfer

Table 6

Correlations of Studied Variables (Study 2 - US Workers)

\begin{tabular}{|c|c|c|c|c|c|c|c|c|c|c|c|c|c|c|c|c|c|c|c|c|c|}
\hline & & $\mathrm{M}$ & SD & 1 & 2 & 3 & 4 & 5 & 6 & 7 & 8 & 9 & 10 & 11 & 12 & 13 & 14 & 15 & 16 & 17 & 18 \\
\hline 1 & LSC & .53 & .50 & & & & & & & & & & & & & & & & & & \\
\hline 2 & Leader effectiveness - T2 & 2.36 & 1.12 & .10 & & & & & & & & & & & & & & & & & \\
\hline 3 & Leader effectiveness $-\mathrm{T} 1$ & 3.54 & 1.28 & .04 & $.25^{* *}$ & & & & & & & & & & & & & & & & \\
\hline 4 & Just treatment $-\mathrm{T} 2$ & 3.31 & 1.08 & .09 & $.65^{* * *}$ & $.28^{* * *}$ & & & & & & & & & & & & & & & \\
\hline 5 & Just treatment $-\mathrm{T} 1$ & 4.04 & 1.11 & .06 & $.19^{*}$ & $.74^{* * *}$ & $.32^{* * *}$ & & & & & & & & & & & & & & \\
\hline 6 & Anxiety (GL) & 2.87 & 1.32 & .04 & -.04 & $-.25^{* *}$ & -.15 & $-.27^{* *}$ & $(.96)$ & & & & & & & & & & & & \\
\hline 7 & Avoidance (GL) & 3.77 & 1.47 & -.02 & -.16 & $-.33^{* * *}$ & $-.23^{* *}$ & $-.27^{* *}$ & $.54^{* * *}$ & $(.97)$ & & & & & & & & & & & \\
\hline 8 & Anxiety (GL) * Avoidance (GL) & 11.86 & 8.52 & .00 & -.04 & $-.18^{*}$ & -.11 & -.06 & .01 & $.59^{* * *}$ & - & & & & & & & & & & \\
\hline 9 & Anxiety (RS) & 2.35 & 1.26 & -.03 & $-.18^{*}$ & $-.42^{* * *}$ & $-.19^{*}$ & $-.53^{* * *}$ & $.42^{* * *}$ & $.23^{* * *}$ & .01 & (.83) & & & & & & & & & \\
\hline 10 & Avoidance (RS) & 3.93 & 1.75 & .02 & $-.23^{* *}$ & $-.70^{* * *}$ & $-.18^{*}$ & $-.66^{* * *}$ & $.34^{* * *}$ & $.58^{* * *}$ & $.31^{* * *}$ & $.41^{* * *}$ & (.94) & & & & & & & & \\
\hline 11 & Anxiety (RS) * Avoidance (RS) & 11.86 & 8.52 & .04 & $-.22^{* *}$ & $-.63^{* * *}$ & $-.18^{*}$ & $-.58^{* * *}$ & .11 & $.37^{* * *}$ & $.39^{* * *}$ & $.23^{*}$ & $.83^{* * *}$ & - & & & & & & & \\
\hline 13 & Conscientiousness & 3.82 & .92 & -.04 & -.04 & $.22^{* *}$ & -.01 & $.18^{*}$ & $-.53^{* * *}$ & $-.35^{* * *}$ & $-.09^{* * *}$ & $-.30^{* * *}$ & $-.30^{* * *}$ & -.13 & .13 & $(.85)$ & & & & & \\
\hline 14 & Extraversion & 2.62 & 1.15 & .16 & .04 & $.21^{*}$ & $.17^{*}$ & $.18^{*}$ & $-.31^{* * *}$ & $-.57^{* * *}$ & $-.42^{* * *}$ & $-.28^{* *}$ & $-.44^{* * *}$ & $-.30^{* * *}$ & $.28^{* *}$ & $.18^{*}$ & $(.90)$ & & & & \\
\hline 15 & Agreeableness & 3.81 & .92 & .06 & .04 & $.28^{* * *}$ & .13 & $.23^{* *}$ & $-.36^{* * *}$ & $-.66^{* * *}$ & $-.35^{* * *}$ & $-.18^{*}$ & $-.42^{* * *}$ & $-.28^{* *}$ & $.33^{* * *}$ & $.26^{* *}$ & $.43^{* * *}$ & $(.88)$ & & & \\
\hline 16 & Neuroticism & 2.36 & .97 & .01 & -.09 & $-.25^{* *}$ & -.14 & $-.23^{* *}$ & $.67^{* * *}$ & $.62^{* * *}$ & $.27^{* * *}$ & $.32^{* * *}$ & $.46^{* * *}$ & $.25^{* *}$ & $-.27^{* * *}$ & $-.40^{* * *}$ & $-.46^{* * *}$ & $-.36^{* * *}$ & $(.82)$ & & \\
\hline 17 & Trait anxiety & 2.06 & .73 & -.06 & -.11 & $-.20^{*}$ & -.11 & $-.19^{*}$ & $.61^{* * *}$ & $.62^{* * *}$ & $.28^{* * *}$ & $.29^{* * *}$ & $.44^{* * *}$ & $.24^{* *}$ & $-.28^{* * *}$ & $-.49^{* * *}$ & $-.45^{* * *}$ & $-.31^{* * *}$ & $.82^{* * *}$ & $(.93)$ & \\
\hline 18 & Relationship importance & 3.00 & 1.26 & .02 & $.21^{*}$ & $.76^{* * *}$ & $.24^{* *}$ & $.68^{* * *}$ & $-.29^{* * *}$ & $-.48^{* * *}$ & $-.27^{* *}$ & $-.33^{* *}$ & $-.84^{* * *}$ & $-.72^{* * *}$ & .07 & $.26^{* *}$ & $.32^{* * *}$ & $.40^{* * *}$ & $-.38^{* * *}$ & $-.33^{* * *}$ & \\
\hline 19 & Daily contact & 3.31 & 1.06 & .16 & .07 & .15 & .09 & .13 & -.14 & $-.23^{* * *}$ & -.13 & -.14 & $-.23^{* *}$ & -.12 & $.26^{* *}$ & $.21^{* *}$ & $.43^{* * *}$ & $.28^{* * *}$ & $-.23^{* *}$ & -.17 & $.26^{* *}$ \\
\hline
\end{tabular}

LSC $=$ Leader similarity condition (low similarity to previous leader $=0$; higher similarity to previous leader $=1$ ); GL $=$ Global attachment; RS $=$ Relationship-specific attachment; att. $=$ attachment ${ }^{* * *} p<.001 ;{ }^{* *} p<.01 ;{ }^{*} p<.05 ; n=137$. 


\section{RUNNING HEAD: Attachment in leader transfer}

Table 7

Correlations of Studied Variables (Study 2 - Indian Workers)

\begin{tabular}{|c|c|c|c|c|c|c|c|c|c|c|c|c|c|c|c|c|c|c|c|c|c|}
\hline & & $\mathrm{M}$ & SD & 1 & 2 & 3 & 4 & 5 & 6 & 7 & 8 & 9 & 10 & 11 & 12 & 13 & 14 & 15 & 16 & 17 & 18 \\
\hline 1 & LSC & .52 & .50 & & & & & & & & & & & & & & & & & & \\
\hline 2 & Leader effectiveness $-\mathrm{T} 2$ & 3.49 & 1.08 & $.35^{* *}$ & - & & & & & & & & & & & & & & & & \\
\hline 3 & Leader effectiveness $-\mathrm{T} 1$ & 3.92 & .90 & -.07 & .17 & - & & & & & & & & & & & & & & & \\
\hline 4 & Just treatment $-\mathrm{T} 2$ & 3.46 & .76 & .15 & $.66^{* * *}$ & .18 & $(.79)$ & & & & & & & & & & & & & & \\
\hline 5 & Just treatment $-\mathrm{T} 1$ & 3.68 & .77 & -.11 & .04 & $.49^{* * *}$ & .18 & $(.77)$ & & & & & & & & & & & & & \\
\hline 6 & Anxiety (GL) & 3.68 & 1.19 & $.26^{* *}$ & $.21^{*}$ & -.13 & .08 & $-.31^{* *}$ & $(.94)$ & & & & & & & & & & & & \\
\hline 7 & Avoidance (GL) & 3.36 & .86 & .09 & -.08 & -.10 & $-.19^{*}$ & $-.32^{* * *}$ & $.24^{*}$ & $(.87)$ & & & & & & & & & & & \\
\hline 8 & Anxiety (GL) * Avoidance (GL) & 12.63 & 5.43 & -.15 & $-.22^{*}$ & .04 & $-.19^{*}$ & .02 & $-.66^{* * *}$ & $.48^{* * *}$ & - & & & & & & & & & & \\
\hline 9 & Anxiety (RS) & 3.44 & 1.61 & .15 & .17 & $-.28^{* *}$ & .06 & $-.34^{* * *}$ & $.69^{* * *}$ & $.28^{* *}$ & $-.36^{* * *}$ & $(.87)$ & & & & & & & & & \\
\hline 10 & Avoidance (RS) & 3.42 & 1.15 & .18 & -.13 & $-.59^{* * *}$ & $-.22^{*}$ & $-.53^{* * *}$ & $.25^{* *}$ & $.55^{* * *}$ & .13 & $.38^{* * *}$ & $(.80)$ & & & & & & & & \\
\hline 11 & Anxiety (RS) * Avoidance (RS) att. & 12.48 & 7.54 & .02 & $-.35^{* * *}$ & $-.31^{* * *}$ & $-.30^{* *}$ & $-.19^{*}$ & $-.42^{* * *}$ & $.22^{*}$ & $.51^{* * *}$ & $-.48^{* * *}$ & $.51^{* * * *}$ & - & & & & & & & \\
\hline 12 & Openness to experiences & 1.86 & .77 & -.17 & -.11 & .06 & -.03 & .04 & $-.48^{* * *}$ & $-.30^{* *}$ & .18 & $-.51^{* * *}$ & $-.25^{* *}$ & $.27^{* *}$ & $(.64)$ & & & & & & \\
\hline 13 & Conscientiousness & 3.56 & .81 & -.12 & -.07 & .16 & .11 & $.24^{*}$ & $-.58^{* * *}$ & $-.34^{* * *}$ & $.20^{*}$ & $-.49^{* * *}$ & $-.35^{* * *}$ & .13 & $.46^{* * *}$ & $(.62)$ & & & & & \\
\hline 14 & Extraversion & 3.23 & .80 & -.02 & .05 & .08 & -.11 & .00 & -.10 & $-.42^{* * *}$ & $-.21^{*}$ & -.04 & $-.28^{* *}$ & -.17 & .16 & .06 & $(.59)$ & & & & \\
\hline 15 & Agreeableness & 3.78 & .77 & -.18 & -.05 & .15 & .09 & .07 & $-.39^{* * *}$ & $-.50^{* * *}$ & -.01 & $-.48^{* * *}$ & $-.33^{* * *}$ & .16 & $.53^{* * *}$ & $.50^{* * *}$ & $.40^{* * * *}$ & $(.73)$ & & & \\
\hline 16 & Neuroticism & 1.17 & .80 & .19 & .19 & -.15 & .04 & $-.43^{* * *}$ & $.63^{* * *}$ & $.23^{*}$ & $-.32^{* * *}$ & $.44^{* * *}$ & $.35^{* * *}$ & -.13 & $-.31^{* * *}$ & $-.46^{* * *}$ & -.08 & $-.31^{* * *}$ & $(.58)$ & & \\
\hline 17 & Trait anxiety & 2.01 & .47 & .16 & .13 & $-.32^{* * *}$ & -.06 & $-.48^{* * *}$ & $.60^{* * *}$ & $.37^{* * *}$ & $-.19^{*}$ & $.49^{* * *}$ & $.43^{* * *}$ & -.05 & $-.42^{* * *}$ & $-.51^{* * *}$ & -.12 & $-.44^{* * *}$ & $.68^{* * * *}$ & $(.78)$ & \\
\hline 18 & Relationship importance & 3.97 & .98 & -.01 & $.20^{*}$ & $.69^{* * *}$ & $.24^{*}$ & $.52^{* * *}$ & -.15 & $-.29^{* *}$ & -.04 & $-.28^{* *}$ & $-.62^{* * *}$ & $-.36^{* * *}$ & .06 & .16 & $.21^{*}$ & .18 & $-.21^{*}$ & $-.41^{* * *}$ & - \\
\hline 19 & Daily contact & 3.81 & .94 & .06 & .02 & $.36^{* * *}$ & -.10 & $.19^{*}$ & -.06 & -.18 & -.10 & -.05 & $-.40^{* * *}$ & $-.23^{*}$ & .15 & $.24^{*}$ & $.36^{* * *}$ & .14 & -.14 & -.18 & $.32^{* * *}$ \\
\hline
\end{tabular}

$\mathrm{LSC}=$ Leader similarity condition (low similarity to previous leader $=0$; higher similarity to previous leader $=1$ ), GL $=\mathrm{Global}$ attachment; RS $=$ Relationship-specific attachment; ${ }^{* * *} p<.001 ;{ }^{* *} p<.01 ;{ }^{*} p<.05 ; n=109$. 
RUNNING HEAD: Attachment in leader transfer

Table 8

Regression Estimates: Predicting Just-Treatment Expectations T2

\begin{tabular}{|c|c|c|c|c|c|}
\hline Variable & Model 1 & Model 2 & Model 3 & \multicolumn{2}{|c|}{ 95\% Confidence Interval } \\
\hline LSC & $-1.823^{* * *}$ & -1.10 & -1.04 & -2.43 & .35 \\
\hline Just treatment $\mathrm{T} 1$ & -.06 & .03 & .01 & -.18 & .20 \\
\hline LSC $*$ Just treatment T1 & $.49^{* * *}$ & $.39^{* *}$ & $.37^{* *}$ & .12 & .62 \\
\hline US culture & & (base) & (base) & & \\
\hline Greek culture & & .14 & .04 & -.97 & 1.05 \\
\hline Indian culture & & 1.33 & 1.06 & -.60 & 2.71 \\
\hline LSC * Greece & & -1.27 & -1.23 & -2.53 & .07 \\
\hline LSC * India & & -1.75 & -1.61 & -3.57 & .35 \\
\hline Anxiety (RS) - T1 & & .02 & .02 & -.21 & .25 \\
\hline Greek * Anxiety (RS) & & .11 & .12 & -.16 & .40 \\
\hline Indian * Anxiety (RS) & & -.31 & -.26 & -.75 & .22 \\
\hline LSC $*$ Anxiety $(\mathrm{RS})$ & & -.12 & -.13 & -.43 & .18 \\
\hline LSC * Greek * Anxiety (RS) & & .28 & .28 & -.09 & .64 \\
\hline LSC * Indian * Anxiety $(\mathrm{RS})$ & & .47 & .44 & -.13 & 1.00 \\
\hline Avoidance (RS) - T1 & & -.13 & -.06 & -.42 & .30 \\
\hline Greek * Avoidance (RS) & & .34 & .33 & -.24 & .90 \\
\hline Indian * Avoidance (RS) & & .65 & .60 & -.13 & 1.32 \\
\hline LSC * Avoidance (RS) & & .24 & .25 & -.17 & .67 \\
\hline LSC * Greek * Avoidance (RS) & & $-.81^{*}$ & $-.81^{*}$ & -1.47 & -.15 \\
\hline LSC * Indian * Avoidance (RS) & & $-.93^{*}$ & $-.91^{*}$ & -1.74 & -.08 \\
\hline Anxious-RS * Avoidance (RS) & & .06 & .06 & -.11 & .23 \\
\hline Greek * Anxious (RS) * Avoidance (RS) & & -.10 & -.10 & -.31 & .10 \\
\hline Indian * Anxious $(\mathrm{RS}) *$ Avoidance $(\mathrm{RS})$ & & -.21 & -.20 & -.46 & .06 \\
\hline LSC $*$ Anxious (RS) $*$ Avoidance (RS) & & -.13 & -.14 & -.33 & .05 \\
\hline LSC * Greek * Anxious (RS) * Avoidance (RS) & & $.29^{*}$ & $.30^{*}$ & .06 & .54 \\
\hline LSC $*$ Indian * Anxious $(\mathrm{RS}) *$ Avoidance $(\mathrm{RS})$ & & .27 & .28 & -.01 & .57 \\
\hline Constant & $3.59^{* * *}$ & $3.02^{* * *}$ & $2.78^{* * *}$ & 1.71 & 3.85 \\
\hline $\mathrm{F}$ & 13.63 & 3.04 & 3.97 & & \\
\hline $\mathrm{R}^{2}$ & $.11^{* * *}$ & $.19^{* * * *}$ & $.20^{* * *}$ & & \\
\hline
\end{tabular}

LSC $=$ Leader similarity condition (low similarity to previous leader $=0$; higher similarity to previous leader $=$

1); RS = Relationship-specific attachment; controlling for trait anxiety, relationship importance and daily

contact in Model 3; (Big-Five personality dimensions did not change results, and are available from the

authors); Confidence Intervals correspond to Model 3; robust $t$-statistics available from the authors; ${ }^{* * *} p<.001$;

${ }^{* *} p<.01 ;{ }^{*} p<.05 ; n=341 ;$ unstandardized coefficients 
Table 9

Test on the Equality of Structural Coefficients of Relationship-Specific Avoidance Attachment across Cultural Groups in the Similar-Leader Condition

\begin{tabular}{|c|c|c|c|c|}
\hline \multirow[b]{2}{*}{ Groups of comparisons } & \multicolumn{2}{|c|}{$\begin{array}{l}\text { Comparison of avoidance attachment } \\
\text { coefficients (no covariates; JT as DV) }\end{array}$} & \multicolumn{2}{|c|}{$\begin{array}{r}\text { Comparison of avoidance attachment coefficients } \\
\text { (JT as DV; all covariates) }\end{array}$} \\
\hline & $d f$ & $\chi^{2}$ & $d f$ & $\chi^{2}$ \\
\hline USA (low CO) vs. GR (high CO) vs. IN (high CO) & 2 & 8.39 & 2 & $10.20^{* * *}$ \\
\hline USA vs. GR & 1 & $\underset{* *}{5.79}$ & 1 & $7.37^{* * *}$ \\
\hline USA vs. IN & 1 & 5.13 & 1 & $6.62^{* *}$ \\
\hline GR vs. IN & 1 & .11 & 1 & .08 \\
\hline
\end{tabular}

${ }^{*} p<.05 ;{ }^{* *} p<.01 ;{ }^{* * *} p<.001 ; \mathrm{JT}=$ Just-treatment expectations; $\mathrm{DV}=$ dependent variable; $\mathrm{CO}=$ collectivistic $; \mathrm{GR}=\mathrm{Greece} ; \mathrm{IN}=\mathrm{India}$. 
RUNNING HEAD: Attachment in leader transfer

Table 10

Test on the Equality of Structural Coefficients of Relationship-Specific Fearful Attachment across Cultural Groups in the Similar-Leader Condition

\begin{tabular}{|c|c|c|c|c|}
\hline \multirow[b]{2}{*}{ Groups of comparisons } & \multicolumn{2}{|c|}{$\begin{array}{r}\text { Comparison of interaction between avoidance and anxious } \\
\text { attachment coefficients (no covariates; JT as DV) }\end{array}$} & \multicolumn{2}{|c|}{$\begin{array}{r}\text { Comparison of avoidance and anxious attachment } \\
\text { coefficients (JT as DV; all covariates) }\end{array}$} \\
\hline & $d f$ & $\chi^{2}$ & $d f$ & $\chi^{2}$ \\
\hline $\begin{array}{r}\text { US (low CO) vs. GR (high CO) } \\
\text { vs. IN (high CO) }\end{array}$ & 2 & $11.17^{* *}$ & 2 & $14.36^{* * *}$ \\
\hline US vs. GR & 1 & $6.71^{* *}$ & 1 & $7.76^{* * *}$ \\
\hline US vs. IN & 1 & $3.71^{\dagger}$ & 1 & $4.70^{*}$ \\
\hline GR vs. IN & 1 & .10 & 1 & .23 \\
\hline
\end{tabular}

${ }^{\dagger} p<.10 ;{ }^{*} p<.05 ;{ }^{* *} p<.01 ;{ }^{* * *} p<.001 ; \mathrm{JT}=$ Just-treatment expectations; DV = dependent variable; CO = collectivistic; GR = Greece; IN = India. 
RUNNING HEAD: Attachment in leader transfer

Table 11

Regression Estimates: Predicting Perceived Leader Effectiveness (T2) by Culture

\begin{tabular}{lrrr}
\hline \multicolumn{1}{c}{ Variable } & United States & Greece & India \\
\hline LSC & -.75 & $-3.84^{* *}$ & $-3.25^{*}$ \\
Leader effectiveness - T1 & $(-1.15)$ & $(-2.70)$ & $(-2.25)$ \\
& -.08 & -.19 & -.25 \\
LSC * Leader effectiveness - T1 & $(-.67)$ & $(1.61)$ & $(-1.31)$ \\
& $.57^{* * *}$ & $.61^{* *}$ & $.74^{* * *}$ \\
Anxiety (GL) & $(4.24)$ & $(3.51)$ & $(3.43)$ \\
& .07 & -.09 & .11 \\
LSC * Anxiety (GL) & $(.49)$ & $(-.43)$ & $(.27)$ \\
& -.11 & $.72^{\dagger}$ & $-.01^{*}$ \\
Avoidance (GL) & $(-.68)$ & $(1.98)$ & $(-.03)$ \\
& $-.56^{* * *}$ & .54 & .13 \\
LSC * Avoidance (GL) & $(-3.88)$ & $(1.35)$ & $(.21)$ \\
& $.64^{* * *}$ & $-.95^{\dagger}$ & -.38 \\
Anxiety (GL) * Avoidance (GL) & $(3.45)$ & $(-1.87)$ & $(-.63)$ \\
& .10 & -.16 & .03 \\
LSC * Anxiety (GL)* Avoidance (GL) & $(1.23)$ & $(-1.36)$ & $(.17)$ \\
& -.08 & $.43^{*}$ & -.06 \\
Constant & $(-.81)$ & $(2.52)$ & $(-.27)$ \\
& $1.60^{* *}$ & $4.5^{* * *}$ & $4.32^{* * *}$ \\
$\mathrm{~N}$ & $(2.61)$ & $(4.43)$ & $(3.08)$ \\
\hline $\mathrm{R}^{2}$ & 137 & 95 & 109 \\
\hline
\end{tabular}

LSC $=$ Leader similarity condition (low similarity to previous leader $=0$; higher similarity to previous leader $=$ 1); GL = Global attachment; ${ }^{* * *} p<.001 ;{ }^{* *} p<.01 ;{ }^{*} p<.05 ; n$ (total) $=341$; controlling for trait anxiety, relationship importance, daily contact and Big Five personality dimensions; robust $t$-statistics in parentheses; unstandardized coefficients. 
RUNNING HEAD: Attachment in leader transfer

Table 12

Test on the Equality of Structural Coefficients of Relationship-Specific Avoidance Attachment across Cultural Groups (DV = LEFF) in the Similar-Leader Condition

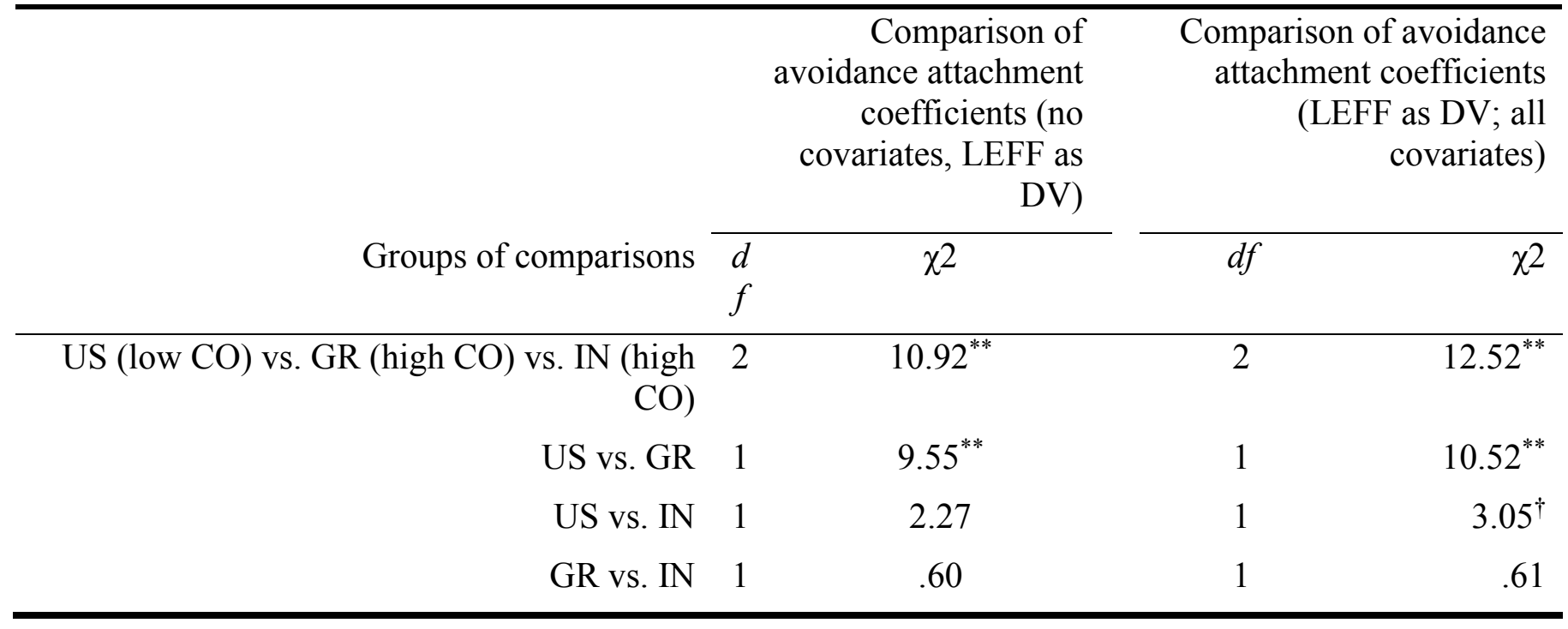

${ }^{*} p<.05,{ }^{* *} p<.01,{ }^{* * *} p<.001 ; \mathrm{LEFF}=$ Leader effectiveness ratings; $\mathrm{DV}=$ dependent

variable; $\mathrm{CO}=$ collectivistic; $\mathrm{US}=$ United States of America; GR = Greece; $\mathrm{IN}=$ India. 
Panel A

Non-Similar Group $(\mathrm{n}=108)$

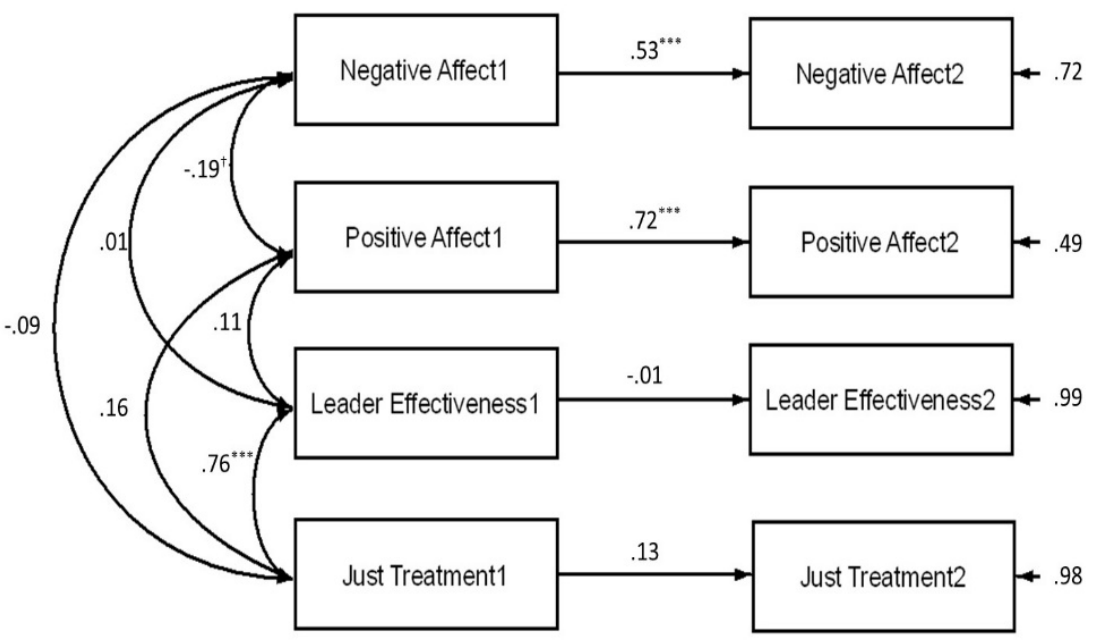

Panel B

Similar Group $(\mathrm{n}=103)$

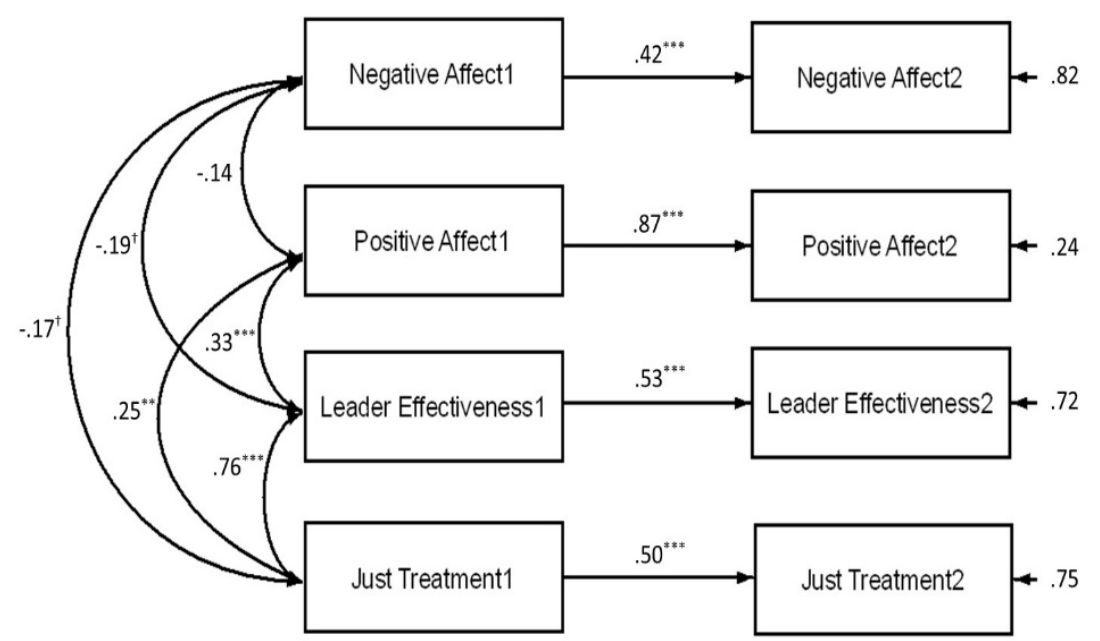

Figure 1 Pre-Test Transference Model Presented Separately for the Non-Similar (A; $n=108)$ and Similar Groups $(B ; n=103)$

Disturbances are correlated. ${ }^{\dagger} p<.10 ;{ }^{*} p<.05 ;{ }^{* *} p<.01 ;{ }^{* * *} p<.001$. 


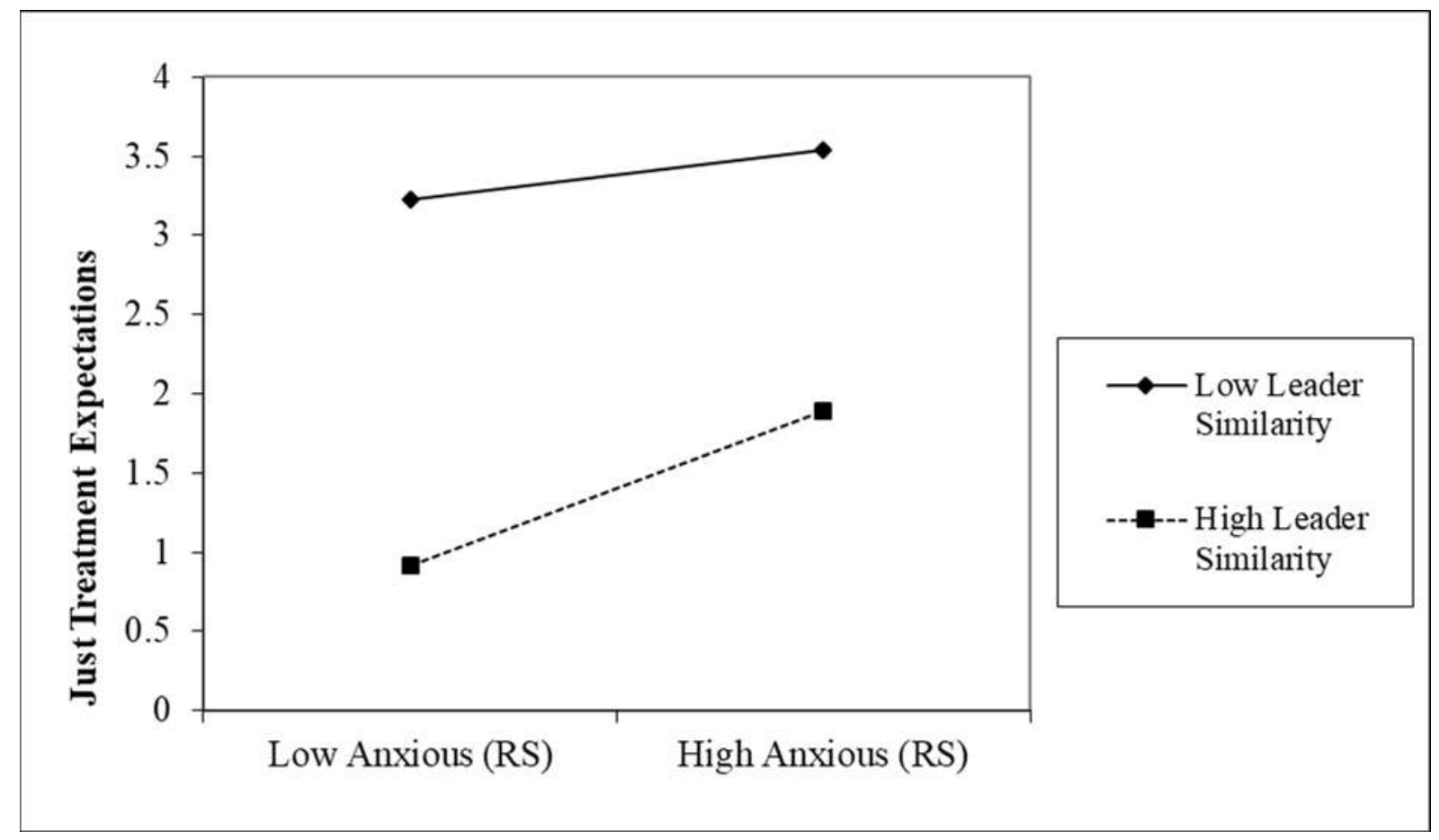

Figure 2 Relationship between Relationship-Specific Anxious Attachment and Just-Treatment Expectations across Conditions (Study 1)

RS = Relationship-specific

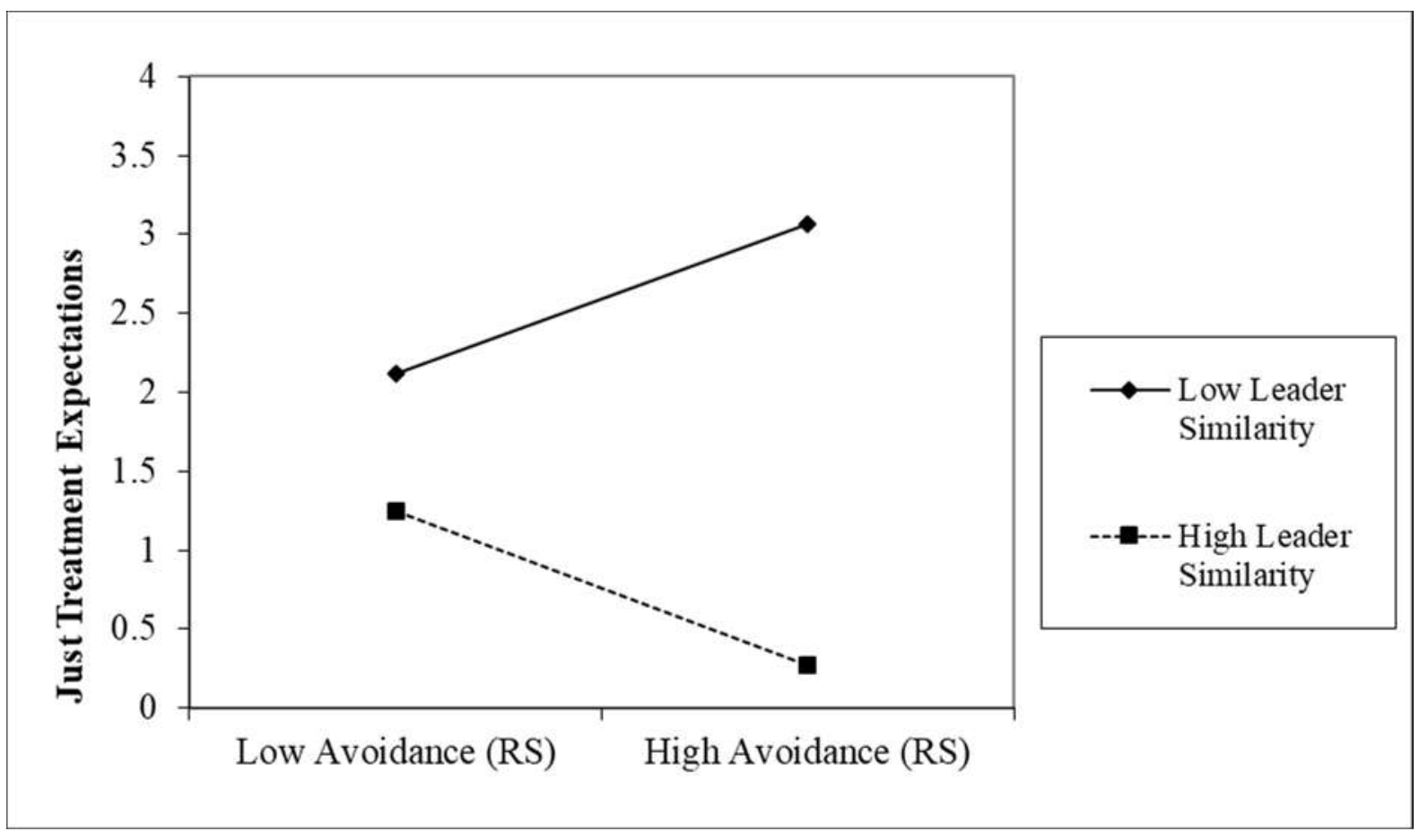

Figure 3 Relationship between Relationship-Specific Avoidant Attachment and Just-Treatment Expectations across Conditions (Study 1); RS = Relationship-specific 


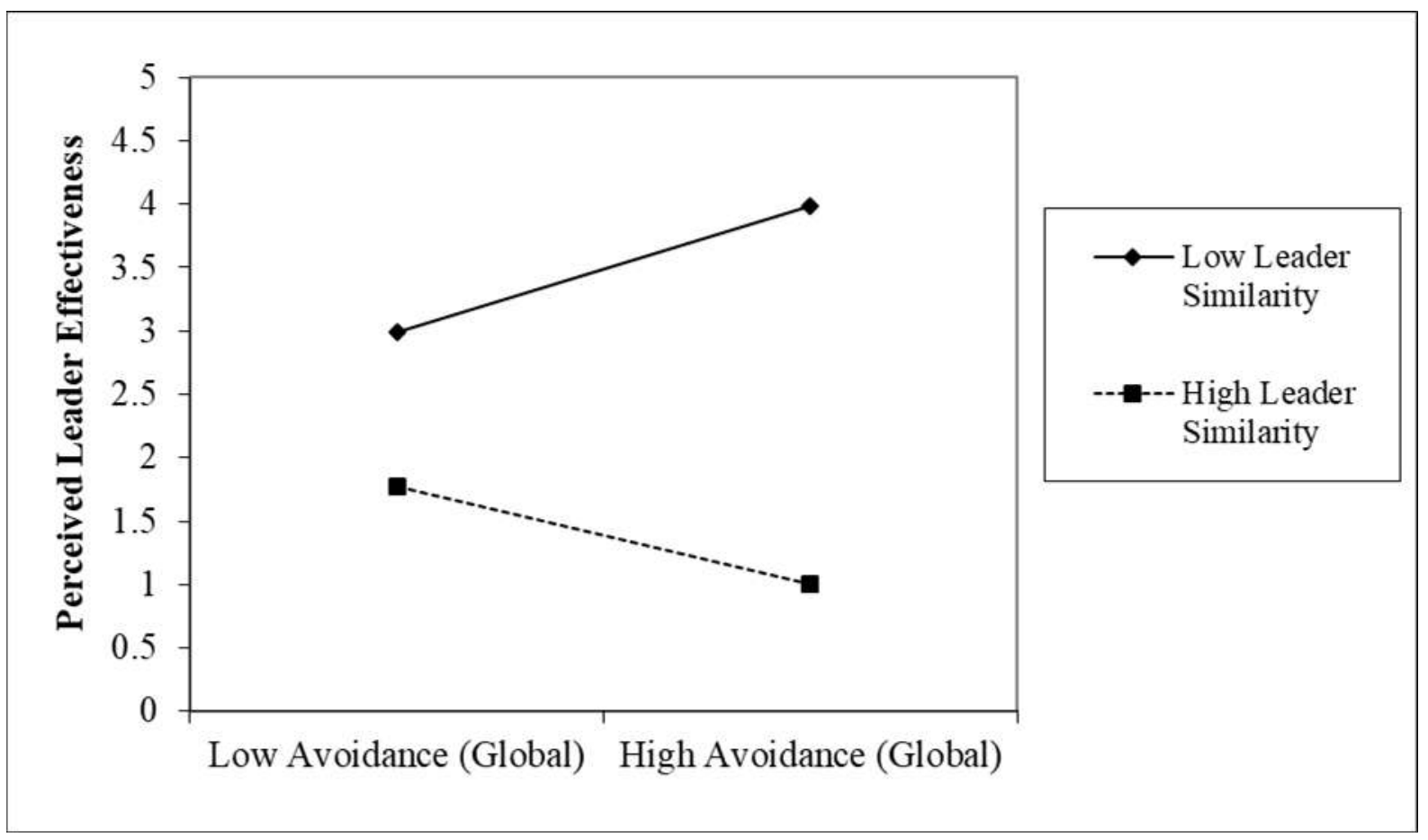

Figure 4 Relationship between Global Avoidant Attachment and Perceived-LeaderEffectiveness Expectations across Conditions (Study 1) 